\title{
Associations between pretreatment physical performance tests and treatment complications in patients with non-small cell lung cancer: A systematic review
}

Citation for published version (APA):

Voorn, M. J. J., Franssen, R. F. W., Verlinden, J. M. W. F., Bootsma, G. P., de Ruysscher, D. K., Bongers, B. C., \& Janssen-Heijnen, M. L. G. (2021). Associations between pretreatment physical performance tests and treatment complications in patients with non-small cell lung cancer: A systematic review. Critical Reviews in Oncology/Hematology, 158. https://doi.org/10.1016/j.critrevonc.2020.103207

Document status and date:

Published: 01/02/2021

DOI:

10.1016/j.critrevonc.2020.103207

Document Version:

Publisher's PDF, also known as Version of record

Document license:

Taverne

Please check the document version of this publication:

- A submitted manuscript is the version of the article upon submission and before peer-review. There can be important differences between the submitted version and the official published version of record. People interested in the research are advised to contact the author for the final version of the publication, or visit the DOI to the publisher's website.

- The final author version and the galley proof are versions of the publication after peer review.

- The final published version features the final layout of the paper including the volume, issue and page numbers.

Link to publication

\footnotetext{
General rights rights.

- You may freely distribute the URL identifying the publication in the public portal. please follow below link for the End User Agreement:

www.umlib.nl/taverne-license

Take down policy

If you believe that this document breaches copyright please contact us at:

repository@maastrichtuniversity.nl

providing details and we will investigate your claim.
}

Copyright and moral rights for the publications made accessible in the public portal are retained by the authors and/or other copyright owners and it is a condition of accessing publications that users recognise and abide by the legal requirements associated with these

- Users may download and print one copy of any publication from the public portal for the purpose of private study or research.

- You may not further distribute the material or use it for any profit-making activity or commercial gain

If the publication is distributed under the terms of Article 25fa of the Dutch Copyright Act, indicated by the "Taverne" license above,

Download date: 26 Apr. 2023 


\title{
Associations between pretreatment physical performance tests and treatment complications in patients with non-small cell lung cancer: A systematic review
}

\author{
M.J.J. Voorn ${ }^{\text {a,b,c, }{ }^{*} \text {, R.F.W. Franssen }}{ }^{\text {a,c, }}{ }^{\text {, J.M.W.F. Verlinden }}{ }^{\mathrm{b}}$, G.P. Bootsma ${ }^{\mathrm{e}}$, D. \\ K. de Ruysscher ${ }^{\mathrm{f}}$, B.C. Bongers ${ }^{\text {g,h }}{ }^{\text {, M.L.G. Janssen-Heijnen }}{ }^{\text {a,c }}$ \\ ${ }^{\text {a }}$ Department of Clinical Epidemiology, VieCuri Medical Center, Venlo, the Netherlands \\ ${ }^{\mathrm{b}}$ Adelante Rehabilitation Center, Venlo, the Netherlands \\ ${ }^{\mathrm{c}}$ Department of Epidemiology, GROW School for Oncology and Developmental Biology, Faculty of Health, Medicine and Life Sciences, Maastricht University, Maastricht, \\ the Netherlands \\ ${ }^{\mathrm{d}}$ Department of Clinical Physical Therapy, VieCuri Medical Center, Venlo, the Netherlands \\ e Department of Pulmonology, Zuyderland Medical Center, Heerlen, the Netherlands \\ ${ }^{\mathrm{f}}$ Department of Radiation Oncology (MAASTRO Clinic), GROW School for Oncology and Developmental Biology, Maastricht University Medical Center, Maastricht, the \\ Netherlands \\ ${ }^{\mathrm{g}}$ Department of Nutrition and Movement Sciences, Nutrition and Translational Research in Metabolism (NUTRIM), Faculty of Health, Medicine and Life Sciences, \\ Maastricht University, Maastricht, the Netherlands \\ ${ }^{\mathrm{h}}$ Department of Epidemiology, Care and Public Health Research Institute (CAPHRI), Faculty of Health, Medicine and Life Sciences, Maastricht University, Maastricht, the \\ Netherlands
}

\section{A R T I C L E I N F O}

\section{Keywords:}

Lung cancer

Perioperative care

Prehabilitation

Preoperative risk assessment

Aerobic capacity

\begin{abstract}
A B S T R A C T
This systematic review evaluated which outcome variables and cut-off values of pretreatment exercise tests are associated with treatment complications in patients with stage I-III non-small cell lung cancer (NSCLC). PRISMA and Cochrane guidelines were followed. A total of 38 studies with adult patients undergoing treatment for stage I-III NSCLC who completed pretreatment exercise tests, and of whom treatment-related complications were recorded were included. A lower oxygen uptake at peak exercise amongst several other variables on the cardiopulmonary exercise test and a lower performance on field tests, such as the incremental shuttle walk test, stair-climb test, and 6-minute walk test, were associated with a higher risk for postoperative complications and/ or postoperative mortality. Cut-off values were reported in a limited number of studies and were inconsistent. Due to the variety in outcomes, further research is needed to evaluate which outcomes and cut-off values of physical exercise tests are most clinically relevant.
\end{abstract}

\section{Introduction}

Lung cancer is the leading cause of cancer-related mortality worldwide, in which non-small cell lung cancer (NSCLC) accounts for $85 \%$ of all lung cancers (Netherlands Cancer Registry, 2016). For fit patients with early stage I, II, and - in some cases - IIIa NSCLC, lung resection is recommended according to European guidelines (Brunelli et al., 2009a). For patients with early stage disease who are considered inoperable, stereotactic radiotherapy is the preferred treatment (Postmus et al., 2017). For fit patients with stage III disease, chemoradiotherapy is the standard treatment with the option of adjuvant immunotherapy after non-progression (Eberhardt et al., 2015). Clinical trials have shown that intensive treatment results in considerably longer disease-free and overall survival in relatively fit patients (Auperin et al., 2010), but is often accompanied with a high incidence of treatment complications (Driessen et al., 2016). Patients with a higher risk for treatment complications are often characterized as aged $\geq 70$ years, having tobacco-related comorbidity and/or cognitive impairment, being physically inactive and/or malnourished, and especially as having a low physiological reserve capacity (low aerobic fitness) (Janssen-Heijnen et al., 2004; Jemal et al., 2011).

When standard pulmonary function tests to verify resectability, such

\footnotetext{
* Corresponding author at: Department of Clinical Epidemiology, VieCuri Medical Center, Tegelseweg 210, 5912 BL, Venlo, the Netherlands.

E-mail address: mvoorn@viecuri.nl (M.J.J. Voorn).
} 
as the forced expiratory volume in $1 \mathrm{~s}\left(\mathrm{FEV}_{1}\right)$ and carbon monoxide lung diffusion capacity (DLCO), fall below $80 \%$ of predicted, a cardiopulmonary exercise test (CPET) is performed for surgical decision-making (Vansteenkiste et al., 2014). Oxygen uptake at peak exercise $\left(\mathrm{VO}_{2 \text { peak }}\right)$ as measured during a CPET has been used most widely for preoperative risk stratification in lung surgery; however, current cut-off values are not based on solid evidence (Warner et al., 2016; Roman et al., 2014). Although the CPET is the gold standard to evaluate a patient's aerobic fitness, it is relatively expensive, time-consuming, and requires trained personnel (Granger et al., 2015; Jones et al., 2007). Hence, practical, cheap, easy to administer, and time efficient field exercise tests such as the incremental shuttle walk test (iSWT), stair-climb test (SCT), 6-minute walk test (6MWT), 12-minute walk test (12MWT), and steep ramp test might be less complicated tests to estimate a patient's preoperative aerobic fitness (Granger et al., 2015; Cavalheri et al., 2016). The use of field exercise tests for estimating aerobic fitness has previously been investigated in patients with cardiac and pulmonary disease (Ambrosino, 1999). Results demonstrated a moderate-to-strong correlation between CPET-derived variables of aerobic fitness and field exercise test outcomes (Holland et al., 2014). Nevertheless, systematic evidence on the association between pretreatment field exercise tests and treatment complications in patients with NSCLC is lacking, especially in patients who undergo chemoradiotherapy.

Due to the predictive value of pretreatment exercise tests for treatment complications, outcome variables of the CPET and field exercise tests might be used to identify high-risk patients who might benefit from lifestyle interventions before and during cancer treatment (prehabilitation and early rehabilitation, respectively). Lifestyle interventions might improve a patient's aerobic fitness, which in turn can improve treatment tolerance and effectiveness (Ni et al., 2017; Perrotta et al., 2019). The aim of this systematic review was to evaluate which outcome variables of pretreatment exercise tests are associated with treatment complications in patients with stage I-III NSCLC, as well as to identify cut-off values for clinical risk stratification.

\section{Methods}

A systematic review was performed with respect to outcome variables of pretreatment exercise tests and their association with treatment complications in patients with stage I-III NSCLC. The Cochrane guidelines for systematic reviews (Vainshelboim, 2019) and the Preferred Reporting Items for Systematic Reviews and Meta-Analyses (PRISMA) guidelines (David Moher et al., 2009) were followed.

\subsection{Literature search}

PubMed, Embase, and Cinahl databases were searched for eligible studies published up to December 2019. In addition, references from retrieved studies were screened. The search strategy contained a combination of controlled vocabulary (e.g., MeSH or EMTREE) and key word terms and phrases searched in titles, abstracts, and key word fields, as appropriate. Key terms included in the search strategy included nonsmall cell lung cancer and lung surgery, exercise test, walk test (6minute walk test and incremental shuttle walk test), cardiopulmonary exercise test or CPET, anaerobic threshold, aerobic fitness, postoperative complications, overall treatment time and postoperative mortality. Combinations of text words of the literature search are shown in Table 1.

\subsection{Study selection}

Prospective and retrospective cohort studies with adult patients undergoing treatment for stage I-III NSCLC who completed pretreatment exercise tests, and of whom treatment-related complications were recorded were included. Studies primarily investigating the impact of prehabilitation or any structured exercise program on physical fitness before treatment, and studies that primarily described survival as outcome measure were excluded. Conference papers, case series, case reports, opinion studies (non-original research), systematic reviews, randomized clinical trials, and studies not published in English were also excluded. Two reviewers (M.V. and R.F.) independently screened titles and abstracts of studies obtained by the literature search. Assessment of

Table 1

Combinations of text words of the literature search according to the PECO-structure.

\begin{tabular}{|c|c|c|c|}
\hline Databases ${ }^{a}$ & Population & Exposure/comparator & Outcome \\
\hline $\begin{array}{l}\text { Embase, } \\
\text { PubMed, } \\
\text { Cinahl }\end{array}$ & $\begin{array}{l}\text { ("lung neoplasms"[MeSH Terms:NoExp] OR "Carcinoma, } \\
\text { Non-Small-Cell Lung"[Mesh] OR lung-neoplasm*[tiab] } \\
\text { OR lung-cancer*[tiab] OR pulmonary-cancer*[tiab] OR } \\
\text { pulmonary-neoplasm*[tiab] OR cancer-of-the-lung* } \\
\text { [tiab] OR cancers-of-the-lung*[tiab] OR non-small-cell- } \\
\text { lung-carcinoma*[tiab] OR NSCLC[tiab] OR non-small- } \\
\text { cell-lung-cancer*[tiab]) AND } \\
\text { ("Chemoradiotherapy"[Mesh] OR } \\
\text { "Radiotherapy"[MeSH] OR "Pulmonary Surgical } \\
\text { Procedures"[MeSH] OR "Pneumonectomy"[Mesh] OR } \\
\text { "Thoracic Surgical Procedures"[MeSH] OR radiation } \\
\text { [tiab] OR radiotherap*[tiab] OR chemotherap*[tiab] } \\
\text { OR radiochemotherapy[tiab] OR radiochemotherapies } \\
\text { [tiab] OR radio-chemotherapy[tiab] OR radio- } \\
\text { chemotherapies[tiab] OR CHRT[tiab] OR } \\
\text { chemoradiation[tiab] OR chemo-radiation[tiab] OR } \\
\text { pulmonary-surgical-procedure*[tiab] OR lung- } \\
\text { operation*[tiab] OR lung-resection*[tiab] OR } \\
\text { ((lobectomy[tiab] OR lobectomies[tiab] OR } \\
\text { segmentectomy[tiab] OR segmentectomies[tiab] OR } \\
\text { resection*[tiab] OR surgery[tiab] OR surgic*[tiab]) } \\
\text { AND (pulmonary*[tiab] OR lung[tiab] OR pneumon* } \\
\text { [tiab])) OR pneumonectomy[tiab] OR thoracic-surgical- } \\
\text { procedure*[tiab] OR "Therapeutics"[Mesh] OR } \\
\text { therapeutic*[tiab] OR treatment*[tiab]) OR operable } \\
\text { [tiab] }\end{array}$ & 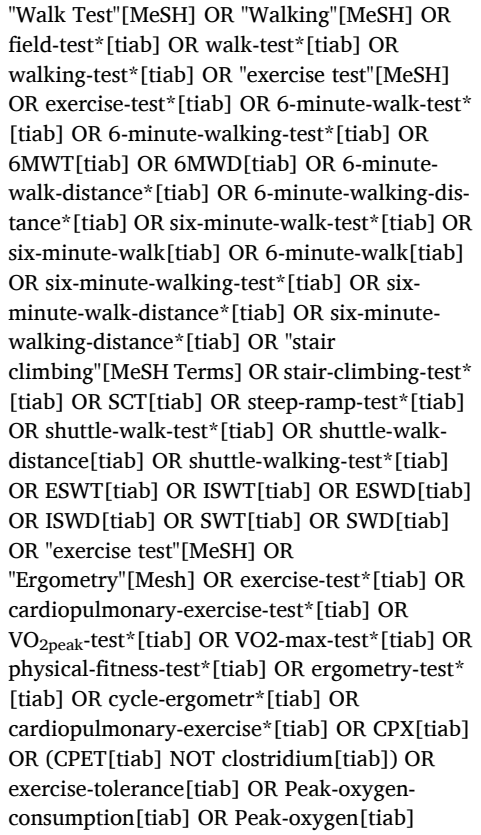 & $\begin{array}{l}\text { "postoperative complications"[MeSH] OR } \\
\text { postoperative-complication*[tiab] OR associated- } \\
\text { conditions[tiab] OR coexistent-disease[tiab] OR } \\
\text { complication*[tiab] OR toxicity-of-side-effects[tiab] } \\
\text { OR toxicit*[tiab] OR adverse-effects[tiab] OR side- } \\
\text { effects[tiab] OR adverse-reaction*[tiab] OR } \\
\text { adverse-events[tiab] OR "mortality"[MeSH] OR } \\
\text { mortality[tiab] OR Mortalities[tiab] OR death[tiab] } \\
\text { OR fatality[tiab] OR fatal*[tiab] OR } \\
\text { "hospitalization"[MeSH] OR hospitalisation[tiab] } \\
\text { OR hospitalization[tiab] OR length-of-stay[tiab] OR } \\
\text { length-of-hospital-stay[tiab] OR patient -discharge } \\
\text { [tiab] OR reduce-treatment-dose[tiab] OR overall- } \\
\text { treatment-time[tiab] OR time-to-treatment[tiab] } \\
\text { OR delay*[tiab] OR dose-modification*[tiab] OR } \\
\text { completion-of-planned-treatment[tiab] OR toxicity- } \\
\text { of-systematic-treatment[tiab] OR withdrawal[tiab] } \\
\text { OR chemotherapy-toxicity[tiab] OR toxicity- } \\
\text { systematic-treatment[tiab] OR postoperative- } \\
\text { decrease[tiab] OR pulmonary function[tiab] OR } \\
\text { health-outcomes[tiab] OR postoperative[tiab] OR } \\
\text { post-operative[tiab] OR operative-risk[tiab] OR } \\
\text { risk-stratification[tiab] }\end{array}$ \\
\hline
\end{tabular}

${ }^{a}$ : search presented for PubMed only: the search strategy has been adjusted for searching in the other databases. 
full texts according to eligibility criteria was performed independently by these two reviewers. Any disagreements between reviewers were resolved through discussion and consensus. When no consensus was reached, a third party acted as an adjudicator (J.V.).

\subsection{Data extraction}

Two authors (M.V. and R.F.) independently extracted data from each of the included studies by using a standardized extraction form. Information collected included the name of the first author, year of publication, type of cohort, sample size, age and sex of participants, used pretreatment exercise test, used test protocol with steps, preselection method, follow-up period, type of cancer treatment, outcome variables of treatment complications, measures for associations between outcomes of pretreatment tests and treatment complications, and cut-off values of pretreatment exercise tests. Complications of treatment were reported as cardiac complications and pulmonary complications or as mortality when mortality was separately identified as a complication.

\subsection{Quality assessment}

The quality of the included studies was assessed using the NewcastleOttawa Scale (NOS) (David Moher et al., 2009). Studies scoring 3 or 4 stars in the selection domain, 1 or 2 stars in the comparability domain, and 2 or 3 stars in the outcome/exposure domain were defined as good-quality studies. Studies scoring 2 stars in the selection domain, 1 or 2 stars in comparability domain, and 2 or 3 stars in outcome/exposure domain were defined as fair-quality studies, and a score of $0-1$ star in selection domain were classified as fair-quality studies. Studies scoring 0 stars in the comparability domain, or 0 or 1 stars in the outcome/exposure domain, were defined as low-quality studies (Wells et al., 2013). Two investigators (M.V. and R.F.) independently assessed the quality of included studies. Discrepancies were resolved by consensus. When consensus was not reached, a third person acted as an adjudicator (J.V.).

\subsection{Data analyses}

Associations between pretreatment exercise tests and treatment complications were interpreted as statistically significant when p-values were $<0.05$. Cut-off values for outcomes of exercise tests for an increased risk of treatment complications were presented when receiver operating characteristic (ROC) curves, including area under the curve (AUC), sensitivity and specificity, and/or odds ratios were determined in the included studies.

\section{Results}

\subsection{Study characteristics}

\subsubsection{Study selection}

Initially, the literature search identified 684 studies, of which 38 were eventually included. A flow diagram for the selection of studies is shown in Fig. 1. An overview of the characteristics of the 38 studies is shown in Table 2. Twenty-three studies were prospective observational, eleven studies were retrospective observational, and four studies had an unclear observational design. The oldest publications dated from 1984 (Smith et al., 1984; Bagg, 1984) and the most recent from 2018 (Kasikcioglu et al., 2018; Yakal et al., 2018; Miyazaki et al., 2018; Nakagawa et al., 2018). Median sample size was 110 patients (ranging from 12 to 287, with a total of 4191) and the mean age of the included patients ranged between 56 and 72 years. In nine studies (24\%), it was indicated which stages of NSCLC had been included (Kasikcioglu et al., 2018; Yakal et al., 2018; Brutsche et al., 2000; Fang et al., 2013;

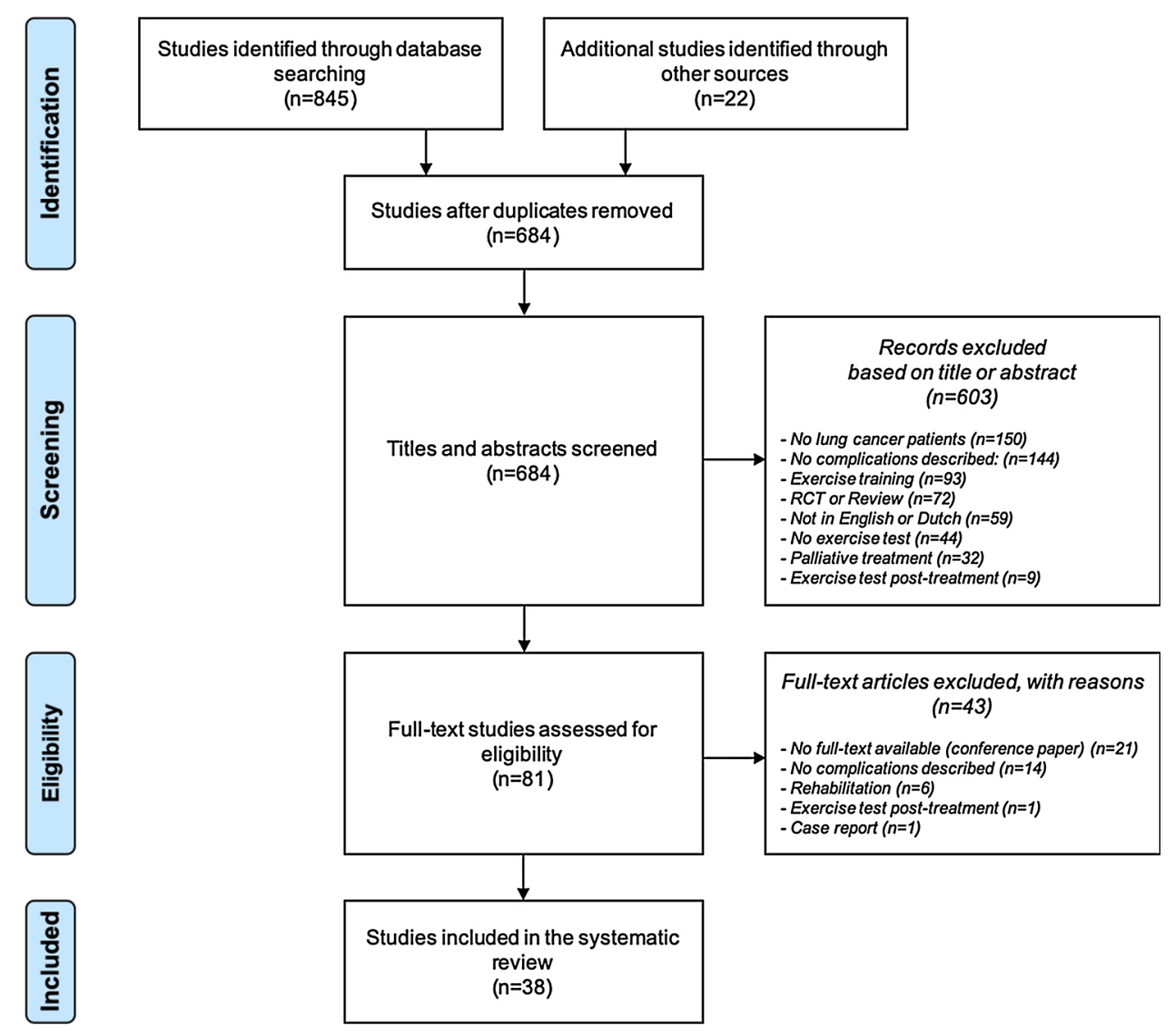

Fig. 1. PRISMA flow diagram displaying the selection of studies and reasons for exclusion. 
Table 2

Study and characteristics of included studies that evaluated the association of preoperative exercise tests and postoperative complications.

\begin{tabular}{|c|c|c|c|c|c|c|c|c|c|}
\hline First author & $\begin{array}{l}\text { Type of } \\
\text { cohort }^{\mathrm{a}}\end{array}$ & $\begin{array}{l}\text { Sample } \\
\text { size (n) }\end{array}$ & $\begin{array}{l}\text { Age (years) } \\
\text { mean } \pm S D \\
\text { (range) }\end{array}$ & $\begin{array}{l}\text { Male } \\
(\%)\end{array}$ & Preselection & $\begin{array}{l}\text { Preoperative } \\
\text { exercise test }\end{array}$ & Protocol & $\begin{array}{l}\text { Follow-up } \\
\text { period } \\
\text { (days) }\end{array}$ & $\begin{array}{l}\text { Type of } \\
\text { surgery }\end{array}$ \\
\hline $\begin{array}{l}\text { (Miyazaki et al., } \\
\text { 2018) }\end{array}$ & Retrospective & 209 & $72.4 \pm 8.3$ & 58 & NR & $\begin{array}{l}\text { CPET (cycle } \\
\text { ergometer) }\end{array}$ & Ramp, $1 \mathrm{~W} / 6 \mathrm{~s}$ & 30,90 & L, S \\
\hline $\begin{array}{l}\text { (Rodrigues } \\
\text { et al., 2016) }\end{array}$ & NR & 54 & $\begin{array}{l}64.7 \pm 7.9 \\
(46-80)\end{array}$ & 92 & NR & $\begin{array}{l}\text { CPET (cycle } \\
\text { ergometer) }\end{array}$ & Ramp, NR & 30 & $\mathrm{~L}$ \\
\hline $\begin{array}{l}\text { Shafiek et al., } \\
\text { 2016) }\end{array}$ & Retrospective & 51 & $65.4 \pm 9.1$ & 82 & $\begin{array}{l}\mathrm{FEV}_{1}<30 \%^{\mathrm{b}} \text { and } \\
\text { DLCO }<40 \%^{\mathrm{b}}\end{array}$ & $\begin{array}{l}\text { CPET (cycle } \\
\text { ergometer) }\end{array}$ & Incremental, NR & 30,365 & $\mathrm{P}, \mathrm{L}, \mathrm{S}$ \\
\hline $\begin{array}{l}\text { (Vargas Fajardo } \\
\text { Mdel et al., } \\
\text { 2014) }\end{array}$ & Prospective & 83 & $\begin{array}{l}64.6 \pm 9.5 \\
(38-80)\end{array}$ & 82 & $\begin{array}{l}\mathrm{FEV}_{1} \text { and } \mathrm{DLCO}< \\
40 \%^{\mathrm{b}}\end{array}$ & $\begin{array}{l}\text { CPET (cycle } \\
\text { ergometer) }\end{array}$ & Ramp, Wasserman & NR & P, L, S, B \\
\hline $\begin{array}{l}\text { (Fang et al., } \\
\text { 2013) }\end{array}$ & Prospective & 107 & $65.3 \pm 7.0$ & 97 & $\mathrm{FEV}_{1}<60 \%{ }^{\mathrm{b}}$ & $\begin{array}{l}\text { CPET (cycle } \\
\text { ergometer) }\end{array}$ & Ramp, $10-20 \mathrm{Watt} / \mathrm{min}$ & 30 & $\mathrm{P}$ \\
\hline $\begin{array}{l}\text { (Licker et al., } \\
\text { 2011) }\end{array}$ & Retrospective & 243 & NR & 58 & $\mathrm{FEV}_{1}<80 \%^{\mathrm{b}}$ & $\begin{array}{l}\text { CPET (cycle } \\
\text { ergometer) }\end{array}$ & Ramp, $20 \mathrm{~W} / \mathrm{min}$ & 30 & $\mathrm{R}$ \\
\hline $\begin{array}{l}\text { (Campione } \\
\text { et al., 2010) }\end{array}$ & Retrospective & 99 & $\begin{array}{l}67.4 \pm 8.1 \\
(41-83)\end{array}$ & 81 & $\mathrm{FEV}_{1} \leq 70 \%{ }^{\mathrm{b}}$ & $\begin{array}{l}\text { CPET (cycle } \\
\text { ergometer) }\end{array}$ & Ramp, $10 \mathrm{~W} / \mathrm{min}$ & 30 & $\mathrm{P}, \mathrm{L}, \mathrm{S}$ \\
\hline $\begin{array}{l}\text { (Varela et al., } \\
\text { 2009) }\end{array}$ & Prospective & 103 & $\begin{array}{l}62.6 \pm 13.5 \\
(20-85)\end{array}$ & NR & NR & $\begin{array}{l}\text { CPET (cycle } \\
\text { ergometer) }\end{array}$ & Incremental, $30 \mathrm{~W} / 2 \mathrm{~min}$ & NR & $\mathrm{P}, \mathrm{L}$ \\
\hline $\begin{array}{l}\text { (Brunelli et al., } \\
\text { 2009b) }\end{array}$ & Prospective & 204 & $66.5 \pm 9.6$ & NR & $\mathrm{FEV}_{1}<30 \%$ b & $\begin{array}{l}\text { CPET (cycle } \\
\text { ergometer) }\end{array}$ & Ramp, NR & 30 & $\begin{array}{l}\text { P, L, S, } \\
\text { WR }\end{array}$ \\
\hline $\begin{array}{l}\text { (Nagamatsu } \\
\text { et al., 2004) }\end{array}$ & NR & 211 & $65.9 \pm 8.4$ & 62 & No preselection & $\begin{array}{l}\text { CPET (cycle } \\
\text { ergometer) }\end{array}$ & Ramp, $20 \mathrm{~W} / 2 \mathrm{~min}$ & 30 & $\mathrm{P}, \mathrm{L}, \mathrm{B}$ \\
\hline $\begin{array}{l}\text { (Villani and } \\
\text { Busia, 2004) }\end{array}$ & NR & 150 & $57.1 \pm 0.7$ & 94 & No preselection & $\begin{array}{l}\text { CPET (cycle } \\
\text { ergometer) }\end{array}$ & Incremental, $25 \mathrm{~W} / 3 \mathrm{~min}$ & 30 & $\mathrm{P}$ \\
\hline $\begin{array}{l}\text { (Villani et al., } \\
\text { 2003) }\end{array}$ & NR & 150 & $\begin{array}{l}57.1 \pm 0.7 \\
(33-79)\end{array}$ & 94 & No preselection & $\begin{array}{l}\text { CPET (cycle } \\
\text { ergometer) }\end{array}$ & Incremental, $25 \mathrm{~W} / 3 \mathrm{~min}$ & 30 & $\mathrm{P}$ \\
\hline $\begin{array}{l}\text { (Brutsche et al., } \\
\text { 2000) }\end{array}$ & Prospective & 125 & $\begin{array}{l}63 \pm 11 \\
(20-80)\end{array}$ & 81 & $\mathrm{FEV}_{1}<1.6 \mathrm{~L}$ & $\begin{array}{l}\text { CPET (cycle } \\
\text { ergometer) }\end{array}$ & Ramp, $20 \mathrm{~W} / \mathrm{min}$ & 30 & $\mathrm{R}$ \\
\hline $\begin{array}{l}\text { (Bechard and } \\
\text { Wetstein, } \\
\text { 1987) }\end{array}$ & Prospective & 50 & $\begin{array}{l}63.8 \\
(47-76)\end{array}$ & 100 & $\begin{array}{l}\mathrm{FEV}_{1}>0.9 \mathrm{~L}, \mathrm{FEV}_{1} \\
\mathrm{WR}>1.2 \mathrm{~L}, \mathrm{FEV}_{1}, \\
\mathrm{P}>1.7 \mathrm{~L}\end{array}$ & $\begin{array}{l}\text { CPET (cycle } \\
\text { ergometer) }\end{array}$ & Incremental, $12.5 \mathrm{~W} / \mathrm{min}$ & 30 & $\mathrm{P}, \mathrm{L}, \mathrm{T}$ \\
\hline $\begin{array}{l}\text { (Bolliger et al., } \\
\text { 1995) }\end{array}$ & Prospective & 25 & $\begin{array}{l}62.8 \pm 8.2 \\
(47-77)\end{array}$ & 68 & $\begin{array}{l}\mathrm{FEV}_{1}<2 \mathrm{~L} \text { and } \\
\mathrm{DLCO}<50 \%{ }^{\mathrm{b}}\end{array}$ & $\begin{array}{l}\text { CPET (cycle } \\
\text { ergometer) }\end{array}$ & Ramp, $20 \mathrm{~W} / \mathrm{min}$ & 30 & NR \\
\hline $\begin{array}{c}\text { (Richter Larsen } \\
\text { et al., 1997) }\end{array}$ & Prospective & 97 & $\begin{array}{l}64.3 \pm 8.9 \\
(38-80)\end{array}$ & 69 & $\mathrm{FEV}_{1}>2.0 \mathrm{~L}$ & $\begin{array}{l}\text { CPET (cycle } \\
\text { ergometer) }\end{array}$ & Ramp, $10-15 \mathrm{Watt} / \mathrm{min}$ & 30 & $\mathrm{P}, \mathrm{L}, \mathrm{S}$ \\
\hline $\begin{array}{l}\text { (Epstein et al., } \\
\text { 1993) }\end{array}$ & Prospective & 42 & $62.7 \pm 2.2$ & 98 & $\mathrm{FEV}_{1}<70 \%{ }^{\mathrm{b}}$ & $\begin{array}{l}\text { CPET (cycle } \\
\text { ergometer) }\end{array}$ & Ramp, Wasserman & 30 & $\mathrm{P}, \mathrm{WR}$ \\
\hline $\begin{array}{l}\text { (Smith et al., } \\
\text { 1984) }\end{array}$ & Prospective & 22 & $55.7 \pm 2.0$ & 86 & No preselection & $\begin{array}{l}\text { CPET (cycle } \\
\text { ergometer) }\end{array}$ & Incremental, $10 \mathrm{~W} / \mathrm{min}$ & 30 & $\mathrm{~L}, \mathrm{~B}, \mathrm{~T}$ \\
\hline $\begin{array}{l}\text { (Pate et al., } \\
\text { 1996) }\end{array}$ & Prospective & 12 & $63.6 \pm 4.9$ & NR & $\mathrm{FEV}_{1}<35 \%{ }^{\mathrm{b}}$ & $\begin{array}{l}\text { CPET (cycle } \\
\text { ergometer), SCT, } \\
\text { 12MWT }\end{array}$ & Incremental, $10 \mathrm{~W} / \mathrm{min}$ & NR & $\mathrm{T}$ \\
\hline $\begin{array}{l}\text { (Holden et al., } \\
\text { 1992) }\end{array}$ & Prospective & 23 & NR & NR & $\mathrm{FEV}_{1}>2.0 \mathrm{~L}$ & $\begin{array}{l}\text { CPET (cycle } \\
\text { ergometer), SCT, } \\
6 \mathrm{MWT}\end{array}$ & Incremental, $15 \mathrm{~W} / \mathrm{min}$ & 30 & $\begin{array}{l}\mathrm{P}, \mathrm{L}, \mathrm{T}, \\
\text { WR }\end{array}$ \\
\hline $\begin{array}{l}\text { (Kasikcioglu } \\
\text { et al., 2018) }\end{array}$ & Prospective & 49 & $\begin{array}{l}61 \pm 9 \\
(35-78)\end{array}$ & 90 & NR & CPET (treadmill) & Naughton & NR & $\begin{array}{l}\mathrm{P}, \mathrm{L}, \mathrm{T} \\
\mathrm{WR}\end{array}$ \\
\hline $\begin{array}{l}\text { (Yakal et al., } \\
\text { 2018) }\end{array}$ & Prospective & 123 & $\begin{array}{l}63 \pm 8 \\
(44-85)\end{array}$ & 85 & No preselection & CPET (treadmill) & Bruce & NR & $\mathrm{P}, \mathrm{L}, \mathrm{WR}$ \\
\hline $\begin{array}{l}\text { (Torchio et al., } \\
\text { 2010) }\end{array}$ & Retrospective & 145 & $\begin{array}{l}64.2 \pm 7.9 \\
(41-82)\end{array}$ & 88 & No preselection & $\begin{array}{l}\text { CPET (cycle } \\
\text { ergometer) }\end{array}$ & Balke & 30 & P, L, S, B \\
\hline $\begin{array}{l}\text { (Win et al., } \\
\text { 2005) }\end{array}$ & Prospective & 99 & $\begin{array}{l}68.4 \pm 8.0 \\
(42-85)\end{array}$ & 60 & No preselection & CPET (treadmill) & Steep & 30 & $\mathrm{P}, \mathrm{L}$ \\
\hline $\begin{array}{l}\text { (Dales et al., } \\
\text { 1993) }\end{array}$ & Retrospective & 117 & $\mathrm{NR}$ & 62 & NR & CPET (treadmill) & Multistage incremental & 30 & $\begin{array}{l}\mathrm{P}, \mathrm{L}, \mathrm{T}, \\
\mathrm{WR}\end{array}$ \\
\hline $\begin{array}{l}\text { (Fennelly et al., } \\
\text { 2016) }\end{array}$ & Retrospective & 101 & $\begin{array}{l}65.5 \pm 11.6 \\
(19-85)\end{array}$ & 32 & $\begin{array}{l}\mathrm{FEV}_{1} \text { and } \mathrm{DLCO}< \\
80 \%^{\mathrm{b}}\end{array}$ & iSWT & Singh & 30 & $\mathrm{~T}$ \\
\hline $\begin{array}{l}\text { (Erdoğan et al., } \\
\text { 2013) }\end{array}$ & Prospective & 24 & $61.5 \pm 8.6$ & 96 & NR & iSWT & Singh & 30 & $\begin{array}{l}\mathrm{P}, \mathrm{L}, \mathrm{B} \\
\mathrm{WR}\end{array}$ \\
\hline $\begin{array}{l}\text { (Win et al., } \\
\text { 2004) }\end{array}$ & Prospective & 111 & $69(42-85)$ & 36 & NR & iSWT & Singh & NR & $\begin{array}{l}\mathrm{P}, \mathrm{L}, \mathrm{B}, \\
\mathrm{WR}\end{array}$ \\
\hline $\begin{array}{l}\text { (Dong et al., } \\
\text { 2017) }\end{array}$ & Retrospective & 171 & $65 \pm 9$ & 76 & NR & SCT & $\begin{array}{l}\text { Symptom-limited: as fast as } \\
\text { they could without stopping } \\
\text { to rest until they reached the } \\
\text { highest floor possible }\end{array}$ & 30 & $\mathrm{~T}$ \\
\hline $\begin{array}{l}\text { (Refai et al., } \\
\text { 2014) }\end{array}$ & Prospective & 287 & $66.5 \pm 8.9$ & 79 & No preselection & SCT & $\begin{array}{l}\text { Climb at a pace of their own } \\
\text { choice, the maximum number } \\
\text { of steps }\end{array}$ & 30 & $\mathrm{P}, \mathrm{L}$ \\
\hline $\begin{array}{l}\text { (Nikolic et al., } \\
\text { 2007) }\end{array}$ & Prospective & 101 & $61.1 \pm 8.4$ & 81 & $\mathrm{FEV}_{1}<2.0 \mathrm{~L}$ & SCT & $\begin{array}{l}\text { Climb the maximum number } \\
\text { of steps, at a pace of their own } \\
\text { choice }\end{array}$ & NR & $\mathrm{P}, \mathrm{K}, \mathrm{T}, \mathrm{B}$ \\
\hline $\begin{array}{l}\text { (Toker et al., } \\
\text { 2007) }\end{array}$ & Prospective & 150 & $60.4 \pm 10.6$ & 85 & NR & SCT & $\begin{array}{l}\text { Do their best during 2-flat } \\
\text { climbing exercises }\end{array}$ & NR & $\mathrm{P}, \mathrm{L}$ \\
\hline
\end{tabular}


Table 2 (continued)

\begin{tabular}{|c|c|c|c|c|c|c|c|c|c|}
\hline First author & $\begin{array}{l}\text { Type of } \\
\text { cohort }^{\mathrm{a}}\end{array}$ & $\begin{array}{l}\text { Sample } \\
\text { size (n) }\end{array}$ & $\begin{array}{l}\text { Age (years) } \\
\text { mean } \pm S D \\
\text { (range) }\end{array}$ & $\begin{array}{l}\text { Male } \\
(\%)\end{array}$ & Preselection & $\begin{array}{l}\text { Preoperative } \\
\text { exercise test }\end{array}$ & Protocol & $\begin{array}{l}\text { Follow-up } \\
\text { period } \\
\text { (days) }\end{array}$ & $\begin{array}{l}\text { Type of } \\
\text { surgery }\end{array}$ \\
\hline $\begin{array}{l}\text { (Brunelli et al., } \\
\text { 2001) }\end{array}$ & Prospective & 115 & $66.5 \pm 9.5$ & 77 & No preselection & SCT & $\begin{array}{l}\text { Symptom-limited: as fast as } \\
\text { they could until they reached } \\
\text { the highest floor possible }\end{array}$ & 30 & $\mathrm{P}, \mathrm{L}$ \\
\hline $\begin{array}{l}\text { (Nakagawa } \\
\text { et al., 2018) }\end{array}$ & Retrospective & 121 & $71.4 \pm 7.0$ & 89 & $\begin{array}{l}\mathrm{FEV}_{1} \text { and } \mathrm{DLCO}< \\
60 \%^{\mathrm{b}}\end{array}$ & $6 \mathrm{MWT}$ & Walking as rapidly as possible & 90 & $\mathrm{~L}, \mathrm{WR}$ \\
\hline $\begin{array}{l}\text { (Irie et al., } \\
\text { 2015) }\end{array}$ & Prospective & 188 & $71(64-77)^{c}$ & 62 & $\begin{array}{l}\text { Tumor } \leq 6 \mathrm{~cm} \text { and } \\
\mathrm{FEV}_{1}>600 \mathrm{~m} \mathrm{~L}\end{array}$ & $6 \mathrm{MWT}$ & ATS statement & NR & $\mathrm{L}$ \\
\hline $\begin{array}{l}\text { (Marjanski } \\
\text { et al., 2015) }\end{array}$ & Retrospective & 253 & 63 & 59 & $\begin{array}{l}\mathrm{FEV}_{1} \text { and } \mathrm{DLCO}< \\
80 \%^{\mathrm{b}}\end{array}$ & $6 \mathrm{MWT}$ & ATS statement & 30,90 & $\mathrm{~L}$ \\
\hline (Ha et al., 2013) & Retrospective & 96 & $65.6 \pm 9.6$ & 52 & NR & 6MWT & ATS statement & 30 & $\mathrm{P}, \mathrm{S}, \mathrm{WR}$ \\
\hline (Bagg (1984) & Prospective & 30 & NR & NR & NR & 12MWT & Cooper & 28 & $\mathrm{~T}$ \\
\hline
\end{tabular}

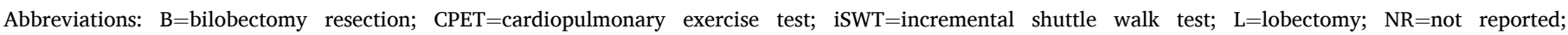

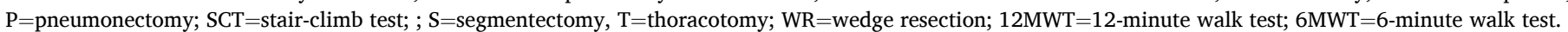

${ }^{\mathrm{a}}$ :all studies were observational. ${ }^{\mathrm{b}}$ :values are expressed as a percentage of predicted. ${ }^{\mathrm{c}}$ :median (interquartile range).

Nagamatsu et al., 2004; Richter Larsen et al., 1997; Ha et al., 2013; Irie et al., 2015; Marjanski et al., 2015), of which five studies (14\%) also reported stage distribution among patients (Campione et al., 2010; Pate et al., 1996; Shafiek et al., 2016; Erdoğan et al., 2013; Win et al., 2004). No study was found in which patients underwent any other NSCLC treatment than surgery, such as chemoradiotherapy. One or more of the following surgical techniques were used in the included studies: pneumonectomy, lobectomy, segmentectomy, bilobectomy, wedge resection, and thoracotomy. Although the initial search strategy captured CPET as well as field exercise tests, the resultant outcomes of the CPET and field exercise tests are presented separately. Preselection of participants by means of $\mathrm{FEV}_{1}$ or DLCO was used in 22 studies (58\%).

\subsubsection{Treatment complications}

In all included studies, surgical resection for NSCLC was performed. Neoadjuvant chemotherapy and/or adjuvant chemotherapy were included in five studies (13\%) (Rodrigues et al., 2016; Brunelli et al., 2009b; Villani and Busia, 2004; Villani et al., 2003; Toker et al., 2007). An association between outcome variables of pretreatment exercise tests and postoperative cardiac and pulmonary complications and/or postoperative mortality was found in 33 of the 38 studies $(87 \%)$. The included studies do not provide information about which complications occur most frequently stratified by type of surgery. The most frequently reported complications were pneumonia (in $88 \%$ of the studies), lobar atelectasis (bronchoscopy required) (78\%), symptomatic cardiac arrhythmias requiring treatment (61\%), myocardial infarction $(60 \%)$, mortality (65\%), pulmonary embolism (57 \%), long-term mechanical ventilation ( $>48 \mathrm{~h}$ ) (51\%), infiltration on chest radiography (27\%), and purulent sputum (19\%). In two studies (5\%), complications were not categorized, and in 15 studies (39\%), postoperative mortality was reported separately.

\subsubsection{Quality assessment}

The results of the quality assessment are depicted in Table 3. In seven studies there was no consensus, because one of the domains was interpreted differently between the reviewers. These discrepancies were resolved by discussion between the two reviewers. In 26 studies (68\%), there was a poor methodological quality, five studies (13\%) were ranked with a fair quality, and seven studies (19\%) had a good quality. A poor score on the Newcastle-Ottawa quality assessment scale was often the result of the lack of: 1) an accurate description of the representativeness of the exposed cohort $(23 / 38,61 \%), 2)$ a clear description of the outcome of interest at start of the study $(34 / 38,89 \%), 3)$ a clear description on the comparability of cases in the cohorts $(21 / 38,55 \%)$, and 4) complete description of complications and/or mortality (24/38, $63 \%$ ). In addition, length of follow-up and adequacy of follow-up of the missing cases were poorly or not described (15/38, $39 \%)$.

\subsection{Pretreatment exercise tests}

Associations between pretreatment exercise tests and postoperative complications are presented in Table 4.

\subsubsection{Cardiopulmonary exercise test}

In $20(80 \%)$ of the 25 studies where the CPET was used preoperatively, one or more outcomes were statistically significant associated with postoperative complications. Cycle ergometry was used in 20 studies (80 \%) (Smith et al., 1984; Miyazaki et al., 2018; Brutsche et al., 2000; Fang et al., 2013; Nagamatsu et al., 2004; Richter Larsen et al., 1997; Campione et al., 2010; Pate et al., 1996; Shafiek et al., 2016; Rodrigues et al., 2016; Vargas Fajardo Mdel et al., 2014; Licker et al., 2011; Varela et al., 2009; Brunelli et al., 2009b; Villani and Busia, 2004; Villani et al., 2003; Bechard and Wetstein, 1987; Bolliger et al., 1995; Epstein et al., 1993; Holden et al., 1992), of which 16 (80\%) reported that preoperative CPET variables were associated with postoperative complications. Different CPET protocols were used, with ten different workload increment protocols. A total of 24 different CPET variables were associated with one or more types of complications after surgery. Fifteen studies (Smith et al., 1984; Miyazaki et al., 2018; Brutsche et al., 2000; Nagamatsu et al., 2004; Richter Larsen et al., 1997; Pate et al., 1996; Shafiek et al., 2016; Rodrigues et al., 2016; Licker et al., 2011; Brunelli et al., 2009b; Villani and Busia, 2004; Villani et al., 2003; Bechard and Wetstein, 1987; Epstein et al., 1993) reported that $\mathrm{VO}_{2 p e a k}$ (both absolute values and values normalized for body mass) was associated with cardiac and pulmonary complications or mortality after surgery, whereas two studies merely reported an association with postoperative pulmonary complications (Fang et al., 2013; Villani et al., 2003). Predicted $\mathrm{VO}_{2 \text { peak }}$ was associated with postoperative cardiac and pulmonary complications (Smith et al., 1984; Brutsche et al., 2000; Fang et al., 2013; Rodrigues et al., 2016; Licker et al., 2011; Villani and Busia, 2004; Villani et al., 2003), pulmonary complications (Brunelli et al., 2009b), and postoperative mortality (Richter Larsen et al., 1997; Brunelli et al., 2009b). Oxygen pulse at peak exercise was found to be associated with postoperative cardiac and pulmonary complications (Fang et al., 2013; Campione et al., 2010; Epstein et al., 1993), as well as with postoperative mortality (Fang et al., 2013). Oxygen uptake at the ventilatory anaerobic threshold normalized for body mass was associated with cardiac and pulmonary complications (Bechard and Wetstein, 1987; Nagamatsu et al., 2015) and postoperative mortality (Fang et al., 2013). The slope describing the relation between minute ventilation and carbon dioxide production ( $\mathrm{VE} / \mathrm{VCO}_{2}$-slope) was also associated with cardiac and pulmonary complications (Miyazaki et al., 2018; Shafiek et al., 2016) and postoperative mortality (Miyazaki et al., 2018). For all associations, a better preoperative score on the respective CPET variable with cycle ergometry was associated with a lower risk of postoperative complications, with the exception of four studies in which no association 
Table 3

Quality assessment based on the Newcastle-Ottawa Scale for cohort studies ${ }^{\text {a }}$.

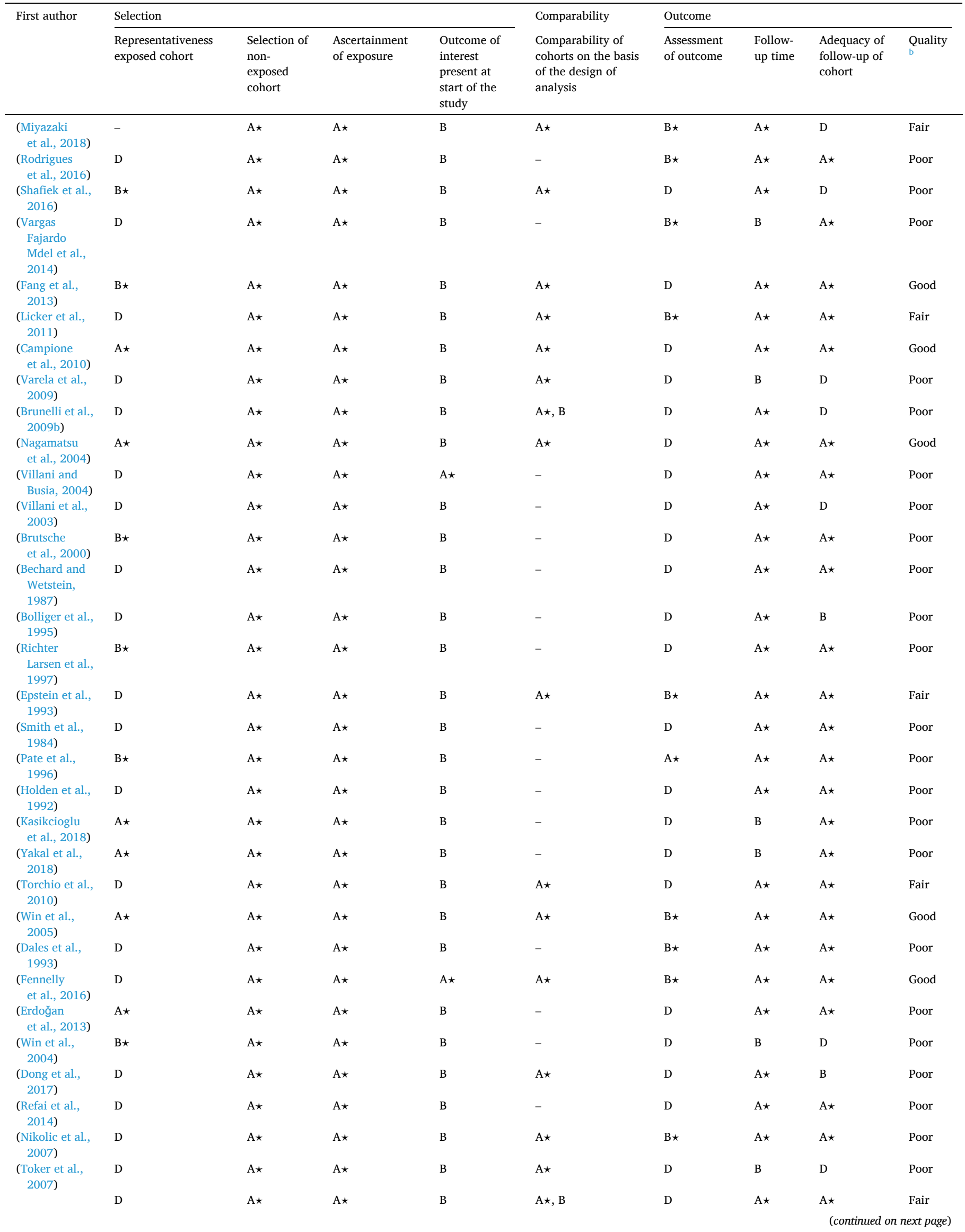


Table 3 (continued)

\begin{tabular}{|c|c|c|c|c|c|c|c|c|c|}
\hline \multirow[t]{2}{*}{ First author } & \multicolumn{4}{|l|}{ Selection } & \multirow{2}{*}{$\begin{array}{l}\text { Comparability } \\
\text { Comparability of } \\
\text { cohorts on the basis } \\
\text { of the design of } \\
\text { analysis }\end{array}$} & \multicolumn{4}{|l|}{ Outcome } \\
\hline & $\begin{array}{l}\text { Representativeness } \\
\text { exposed cohort }\end{array}$ & $\begin{array}{l}\text { Selection of } \\
\text { non- } \\
\text { exposed } \\
\text { cohort }\end{array}$ & $\begin{array}{l}\text { Ascertainment } \\
\text { of exposure }\end{array}$ & $\begin{array}{l}\text { Outcome of } \\
\text { interest } \\
\text { present at } \\
\text { start of the } \\
\text { study }\end{array}$ & & $\begin{array}{l}\text { Assessment } \\
\text { of outcome }\end{array}$ & $\begin{array}{l}\text { Follow- } \\
\text { up time }\end{array}$ & $\begin{array}{l}\text { Adequacy of } \\
\text { follow-up of } \\
\text { cohort }\end{array}$ & $\begin{array}{l}\text { Quality } \\
\text { b }\end{array}$ \\
\hline \multicolumn{10}{|l|}{$\begin{array}{l}\text { (Brunelli et al., } \\
\text { 2001) }\end{array}$} \\
\hline $\begin{array}{l}\text { (Nakagawa } \\
\text { et al., 2018) }\end{array}$ & $\mathrm{D}$ & $A \star$ & $A \star$ & $A \star$ & - & $\mathrm{B} \star$ & $A \star$ & B & Poor \\
\hline $\begin{array}{l}\text { (Irie et al., } \\
\text { 2015) }\end{array}$ & $\mathrm{B} \star$ & $A \star$ & $A \star$ & $A \star$ & - & $\mathrm{B} \star$ & B & $A \star$ & Good \\
\hline $\begin{array}{l}\text { (Marjanski } \\
\text { et al., 2015) }\end{array}$ & $\mathrm{B} \star$ & $\mathrm{A} \star$ & $A \star$ & B & $A \star$ & $\mathrm{B} \star$ & $A \star$ & $A \star$ & Good \\
\hline $\begin{array}{l}\text { (Ha et al., } \\
\text { 2013) }\end{array}$ & $A \star$ & $A \star$ & $A \star$ & B & - & $\mathrm{B} \star$ & $A \star$ & $A \star$ & Poor \\
\hline (Bagg (1984) & $\mathrm{D}$ & $A \star$ & $A \star$ & B & - & $\mathrm{D}$ & $A \star$ & $\mathrm{D}$ & Poor \\
\hline
\end{tabular}

a stars $(\star)$ are awarded on the basis of answers (A, B, C, or D) provided for each item.

b thresholds for converting the Newcastle-Ottawa scale scores to AHRQ standards (good, fair, and poor): good quality = 3 or 4 stars in the selection domain AND 1 or

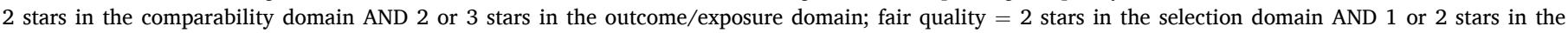

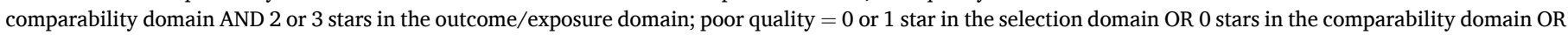
0 or 1 stars in the outcome/exposure domain.

was reported (20 \%) (Vargas Fajardo Mdel et al., 2014; Varela et al., 2009; Bolliger et al., 1995; Holden et al., 1992). In five studies (20 \%) (Kasikcioglu et al., 2018; Yakal et al., 2018; Torchio et al., 2010; Win et al., 2005; Dales et al., 1993) treadmill ergometry was performed. In these studies, a total of seven different CPET outcomes were associated with one or more types of postoperative complications and/or postoperative mortality. Absolute $\mathrm{VO}_{2 \text { peak }}$ was associated with postoperative cardiac and pulmonary complications (Kasikcioglu et al., 2018; Yakal et al., 2018; Torchio et al., 2010) with pulmonary complications (Dales et al., 1993), and postoperative mortality (Yakal et al., 2018; Torchio et al., 2010). Predicted $\mathrm{VO}_{2 \text { peak }}$ was associated with postoperative complications (Win et al., 2005). The oxygen uptake efficiency slope (Kasikcioglu et al., 2018; Yakal et al., 2018) and the VE/ $\mathrm{VCO}_{2}$-slope were associated with cardiac and pulmonary complications after surgery (Torchio et al., 2010).

\subsubsection{Incremental shuttle walk test}

Three studies (Erdoğan et al., 2013; Win et al., 2004; Fennelly et al., 2016) investigated the association between preoperative iSWT performance and postoperative complications. One study reported that oxygen desaturation $\geq 4 \%$ during the iSWT, and distance walked $<400 \mathrm{~m}$ were associated with a higher risk of postoperative complications (Fennelly et al., 2016). In two studies, no associations were found between outcomes of the preoperative iSWT and postoperative complications (Erdoğan et al., 2013; Win et al., 2004).

\subsubsection{Stair-climb test}

A preoperative SCT was performed in seven studies (Pate et al., 1996; Holden et al., 1992; Dong et al., 2017; Refai et al., 2014; Nikolic et al., 2007; Toker et al., 2007; Brunelli et al., 2001), in which different SCT protocols were used. Patients were asked 1) to climb the maximum number of steps at a pace of their own choice (Pate et al., 1996; Holden et al., 1992; Refai et al., 2014; Nikolic et al., 2007; Brunelli et al., 2001), 2) to climb five stairs with 20 steps as fast as they could without stopping to rest (Dong et al., 2017), or 3) to do their best during 2 stair-climbing exercises in which each flight of stairs was composed of 20 steps and climbing time was recorded (Toker et al., 2007). There were also differences between studies concerning test duration, step height, and number of steps. The total number of steps that were taken was associated with postoperative complications (Pate et al., 1996; Brunelli et al., 2009b; Holden et al., 1992; Nikolic et al., 2007) and postoperative mortality (Nikolic et al., 2007). There was an association between the height of climbing in meters, exercise oxygen desaturation, and the change in heart rate from start to finish on the one hand and cardiac and pulmonary complications after surgery on the other hand (Dong et al., 2017). Test duration, speed, heart rate, and oxygen saturation during exercise were associated with postoperative complications and postoperative mortality (Nikolic et al., 2007). Oxygen saturation at the end of the SCT, and the change in oxygen saturation during the SCT were associated with postoperative complications (Toker et al., 2007). In all studies where the preoperative SCT was used, better scores on the test variables were associated with a lower risk of postoperative complications, with the exception of one study that reported no association (Pate et al., 1996).

\subsubsection{Six- and twelve-minute walk test}

Five studies (Nakagawa et al., 2018; Ha et al., 2013; Irie et al., 2015; Marjanski et al., 2015; Holden et al., 1992) assessed the ability of the preoperative $6 \mathrm{MWT}$ to predict the risk of postoperative complications and postoperative mortality. Distance walked as a percentage of predicted was associated with cardiac and pulmonary complications ( $\mathrm{Ha}$ et al., 2013). Other studies reported an association between shorter walked distances and a higher risk of postoperative complications (Irie et al., 2015; Marjanski et al., 2015; Pate et al., 1996; Holden et al., 1992), and postoperative mortality (Holden et al., 1992). All studies using the preoperative 6MWT showed that a poor performance was associated with a higher risk for postoperative complications. Two studies (Bagg, 1984; Pate et al., 1996) used the 12MWT during the preoperative assessment. Both studies reported no association between the distance walked and postoperative complications. One small study (Pate et al., 1996) described a relation between the walked distance in meters and complications, in which a better performance on the 12MWT was associated with a lower risk on postoperative complications.

\subsubsection{Cut-off values}

Cut-off values of outcomes of pretreatment exercise tests associated with an increased risk of postoperative complications and postoperative mortality are presented in Table 5. A limited number of studies reported a cut-off value of outcomes of pretreatment exercise tests for a higher risk for postoperative complications; however, the accuracy of these cutoff values was usually moderate. A study using the CPET on a cycle ergometer reported $\mathrm{VO}_{2 \text { peak }}$ cut-off values of $<12.8 \mathrm{~mL} / \mathrm{kg} / \mathrm{min}$ and $<58$ $\%$ of predicted to be optimal cut-off values for a higher risk for postoperative cardiac complications (Licker et al., 2011). In the same study, optimal cut-off values indicating a higher risk for postoperative pulmonary complications were a $\mathrm{VO}_{2 \text { peak }}<13.6 \mathrm{~mL} / \mathrm{kg} / \mathrm{min}$ or a predicted 
Table 4

Association between preoperative exercise tests and postoperative complications.

\begin{tabular}{|c|c|c|c|c|c|c|c|c|c|}
\hline \multirow{2}{*}{$\begin{array}{l}\text { First author } \\
\text { Cardiopulmonary e }\end{array}$} & \multirow{2}{*}{$\begin{array}{l}\text { Mean age of patients } \\
\text { without / with } \\
\text { complications (years) }\end{array}$} & \multicolumn{4}{|c|}{$\begin{array}{l}\text { Variables associated with cardiac and/or pulmonary } \\
\text { complications }\end{array}$} & \multicolumn{4}{|c|}{ Variables associated with postoperative mortality } \\
\hline & & $\mathrm{VO}_{2 \text { peak }}$ & $\begin{array}{l}\mathrm{VE} / \\
\mathrm{VCO}_{2^{-}} \\
\text {slope }\end{array}$ & $\begin{array}{l}\mathrm{VO}_{2 \text { peak }}(\% \text { of } \\
\text { predicted })\end{array}$ & Other & $\mathrm{VO}_{2 \text { peak }}$ & $\begin{array}{l}\mathrm{VE} / \\
\mathrm{VCO}_{2^{-}} \\
\text {slope }\end{array}$ & $\begin{array}{l}\mathrm{VO}_{2 p e a k}(\% \text { of } \\
\text { predicted) }\end{array}$ & Other \\
\hline $\begin{array}{l}\text { (Miyazaki et al., } \\
\text { 2018) }\end{array}$ & NR & $\mathrm{Y}$ & $\mathrm{Y}$ & - & - & - & $\mathrm{Y}$ & - & - \\
\hline $\begin{array}{l}\text { (Rodrigues et al., } \\
\text { 2016) }\end{array}$ & $65.0 / 64.1$ & $\mathrm{Y}$ & - & $\mathrm{Y}$ & - & $\mathrm{N}$ & - & - & - \\
\hline $\begin{array}{l}\text { (Shafiek et al., } \\
\text { 2016) }\end{array}$ & $64.0 / 67.1$ & $\mathrm{Y}$ & $\mathrm{Y}$ & & $\mathrm{WR}_{\text {peak }}$ & - & - & - & - \\
\hline $\begin{array}{l}\text { (Vargas Fajardo } \\
\text { Mdel et al., } \\
\text { 2014) }\end{array}$ & $63.8 / 69.0$ & $\mathrm{~N}$ & - & $\mathrm{N}$ & - & - & - & & - \\
\hline (Fang et al., 2013) & $64.7 / 66.9$ & $\mathrm{Y}$ & - & $\mathrm{Y}$ & $\begin{array}{l}\mathrm{O}_{2} \text { pulse }_{\text {peak }} \\
\Delta \mathrm{SpO}_{2} \\
\text { VAT }\end{array}$ & - & - & - & - \\
\hline $\begin{array}{l}\text { (Licker et al., } \\
\text { 2011) }\end{array}$ & $62 / 66$ & $\mathrm{Y}$ & - & $\mathrm{Y}$ & - & - & - & - & - \\
\hline $\begin{array}{l}\text { (Campione et al., } \\
\text { 2010) }\end{array}$ & $67.2 / 68.3$ & $\mathrm{~N}$ & - & - & $\mathrm{O}_{2}$ pulse $_{\text {peak }}$ & - & - & - & - \\
\hline $\begin{array}{l}\text { (Varela et al., } \\
\text { 2009) }\end{array}$ & NR & - & - & - & - & - & - & - & - \\
\hline $\begin{array}{l}\text { (Brunelli et al., } \\
\text { 2009b) }\end{array}$ & $66.3 / 67.6$ & $\mathrm{Y}$ & - & $\mathrm{Y}^{\mathrm{b}}$ & - & $\mathrm{Y}$ & - & $\mathrm{Y}$ & $\mathrm{O}_{2}$ pulse $_{\text {peak }}$ \\
\hline $\begin{array}{l}\text { (Nagamatsu et al., } \\
\text { 2004) }\end{array}$ & NR & $\mathrm{Y}$ & - & - & VAT & - & - & - & - \\
\hline $\begin{array}{l}\text { (Villani and } \\
\text { Busia, 2004) }\end{array}$ & $57.2 / 57.1$ & $\mathrm{Y}$ & - & $\mathrm{Y}$ & $\mathrm{WR}_{\text {peak }}$ & - & - & - & - \\
\hline $\begin{array}{l}\text { (Villani et al., } \\
\text { 2003) }\end{array}$ & $57.2 / 57.1$ & $\mathrm{Y}$ & - & $\mathrm{Y}$ & $\mathrm{WR}_{\text {peak }}$ & - & - & - & - \\
\hline $\begin{array}{l}\text { (Brutsche et al., } \\
\text { 2000) }\end{array}$ & $63 / 64$ & $\mathrm{Y}$ & - & $\mathrm{Y}$ & - & - & - & - & - \\
\hline $\begin{array}{l}\text { (Bechard and } \\
\text { Wetstein, 1987) }\end{array}$ & $63.6 / 66.6$ & $\mathrm{Y}$ & - & - & VAT & - & - & - & - \\
\hline $\begin{array}{l}\text { (Bolliger et al., } \\
\text { 1995) }\end{array}$ & NR & $\mathrm{N}$ & - & $\mathrm{N}$ & - & - & - & - & - \\
\hline $\begin{array}{l}\text { (Richter Larsen } \\
\text { et al., 1997) }\end{array}$ & NR & $\mathrm{Y}$ & - & - & $\begin{array}{l}W R_{\text {peak }} \\
\mathrm{VE}_{\text {peak }}\end{array}$ & - & - & $\mathrm{Y}$ & $\mathrm{WR}_{\text {peak }}$ \\
\hline $\begin{array}{l}\text { (Epstein et al., } \\
\text { 1993) }\end{array}$ & $63 / 62$ & $\mathrm{Y}$ & - & - & $\mathrm{O}_{2}$ pulse $_{\text {peak }}$ & $\mathrm{Y}$ & - & - & - \\
\hline $\begin{array}{l}\text { (Smith et al., } \\
\text { 1984) }\end{array}$ & $51.8 / 59.6$ & $\mathrm{Y}$ & - & $\mathrm{Y}$ & - & - & - & - & - \\
\hline (Pate et al., 1996) & $64.2 / 63.1$ & $\mathrm{Y}$ & - & - & - & - & - & - & - \\
\hline $\begin{array}{l}\text { (Holden et al., } \\
\text { 1992) }\end{array}$ & $67.0 / 70.1$ & $\mathrm{~N}$ & - & - & - & $\mathrm{N}$ & - & - & - \\
\hline Cardioulmonary ex & rcise test, treadmill & $\mathrm{VO}_{2 \text { peak }}$ & $\begin{array}{l}\mathrm{VE} / \\
\mathrm{VCO}_{2^{-}} \\
\text {slope }\end{array}$ & $\begin{array}{l}\mathrm{VO}_{2 \text { peak }}(\% \text { of } \\
\text { predicted) }\end{array}$ & Other & $\mathrm{VO}_{2 \text { peak }}$ & $\begin{array}{l}\mathrm{VE} / \\
\mathrm{VCO}_{2-} \\
\text { slope }\end{array}$ & $\begin{array}{l}\mathrm{VO}_{2 \text { peak }}(\% \text { of } \\
\text { predicted) }\end{array}$ & Other \\
\hline $\begin{array}{l}\text { (Kasikcioglu } \\
\text { et al., 2018) }\end{array}$ & NR & $\mathrm{Y}$ & - & - & OUES & - & - & - & - \\
\hline $\begin{array}{l}\text { (Yakal et al., } \\
\text { 2018) }\end{array}$ & NR & $\mathrm{Y}$ & - & - & $\begin{array}{l}\text { OUES } \\
\text { VE }_{\text {peak }} \\
\text { HR at the VAT }\end{array}$ & $\mathrm{Y}$ & - & - & $\begin{array}{l}\text { OUES } \\
\text { VE }_{\text {peak }}\end{array}$ \\
\hline $\begin{array}{l}\text { (Torchio et al., } \\
\text { 2010) }\end{array}$ & $63.7 / 67.1$ & $\mathrm{Y}$ & $\mathrm{Y}$ & $\mathrm{N}$ & - & $\mathrm{Y}$ & $\mathrm{Y}$ & $\mathrm{N}$ & - \\
\hline (Win et al., 2005) & NR & $\mathrm{N}$ & - & $\mathrm{Y}$ & - & $\mathrm{N}$ & - & $\mathrm{N}$ & - \\
\hline $\begin{array}{l}\text { (Dales et al., } \\
\text { 1993) }\end{array}$ & NR & $\mathrm{Y}^{\mathrm{b}}$ & - & $\mathrm{N}$ & $\mathrm{VE}_{\text {peak }} \mathrm{b}$ & - & - & - & - \\
\hline Incremental shuttle & walk test & Distance & & & & & & & \\
\hline $\begin{array}{l}\text { (Fennelly et al., } \\
\text { 2016) }\end{array}$ & $64.0 / 70.7$ & $\mathrm{Y}$ & - & - & - & - & - & - & - \\
\hline $\begin{array}{l}\text { (Erdoğan et al., } \\
\text { 2013) }\end{array}$ & NR & $\mathrm{N}$ & - & - & - & - & - & - & - \\
\hline (Win et al., 2004) & NR & $\mathrm{N}$ & - & - & - & - & - & & - \\
\hline Stair-climb test & & $\begin{array}{l}\text { Height of } \\
\text { climbing }\end{array}$ & Steps & & Other & $\begin{array}{l}\text { Height of } \\
\text { climbing }\end{array}$ & Steps & & Other \\
\hline $\begin{array}{l}\text { (Dong et al., } \\
\text { 2017) }\end{array}$ & NR & $\mathrm{Y}$ & - & - & $\begin{array}{l}\text { Predicted } \\
\text { exercise } \mathrm{SpO}_{2} \\
\Delta \mathrm{HR}\end{array}$ & - & - & - & - \\
\hline $\begin{array}{l}\text { (Refai et al., } \\
\text { 2014) }\end{array}$ & $65.5 / 69.7$ & $\mathrm{~N}$ & - & - & - & - & - & - & - \\
\hline $\begin{array}{l}\text { (Nikolic et al., } \\
\text { 2007) }\end{array}$ & $58.2 / 67.1$ & - & $\mathrm{Y}$ & - & & - & $\mathrm{Y}$ & - & $\begin{array}{l}\mathrm{SpO}_{2} \\
\text { during }\end{array}$ \\
\hline
\end{tabular}


Table 4 (continued)

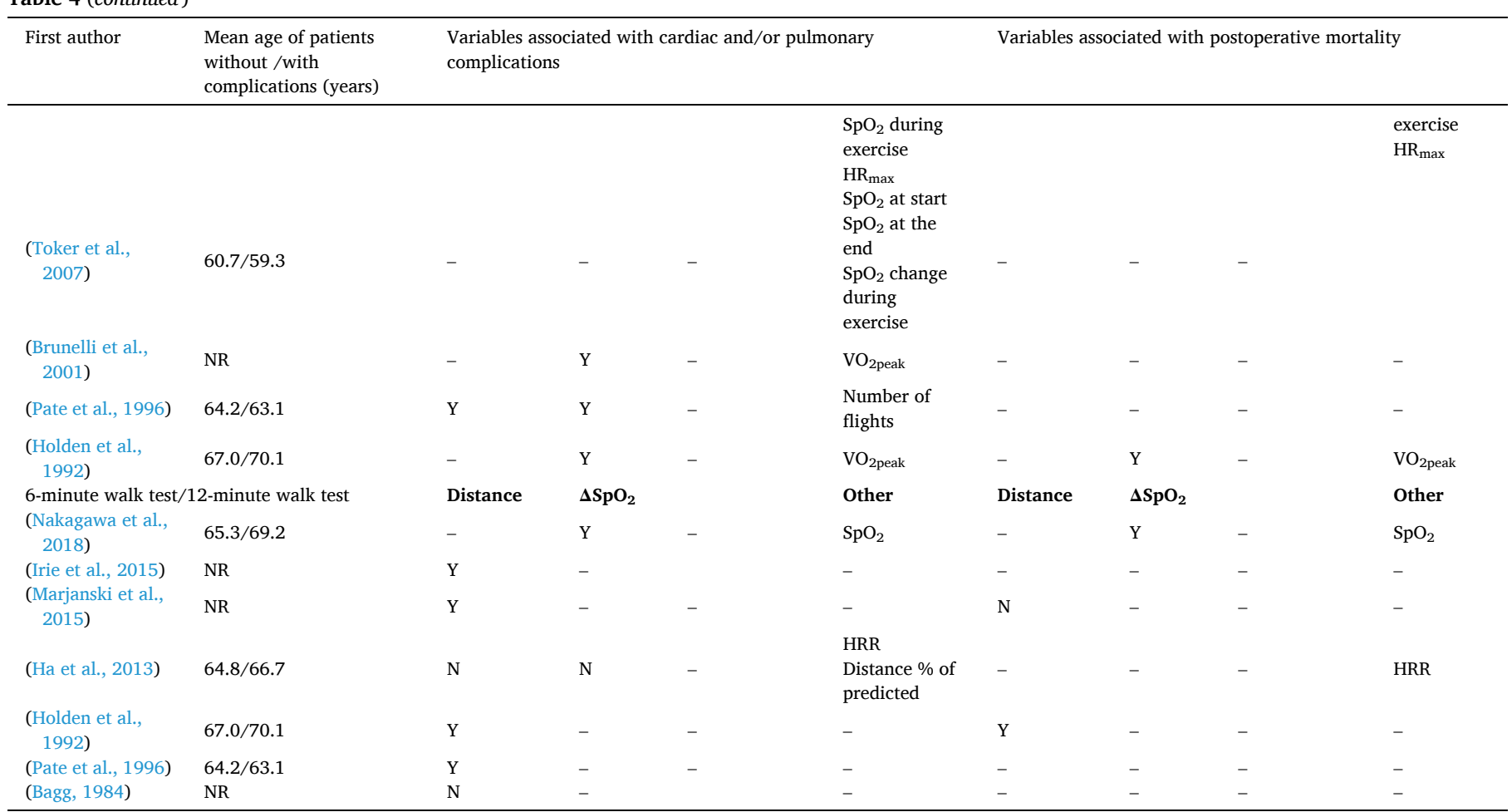

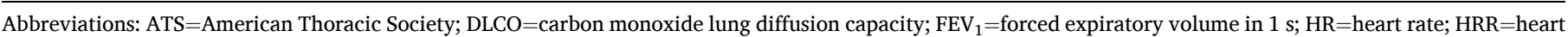

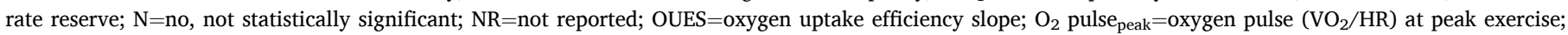

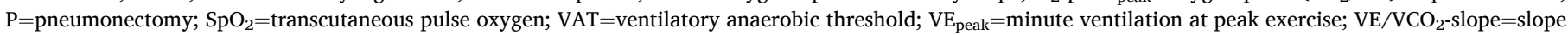

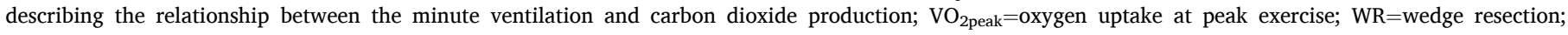

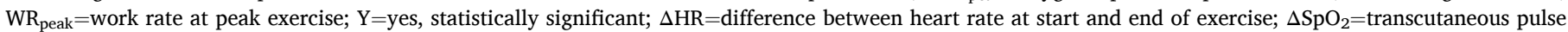
oxygen saturation difference during load exercise.

$\mathrm{a}_{\%} \%$ of predicted.

${ }^{\mathrm{b} O n l y}$ pulmonary complications.

$\mathrm{VO}_{2 \text { peak }}<53 \%$ of predicted, whereas a $\mathrm{VO}_{2 \text { peak }}<12.3 \mathrm{~mL} / \mathrm{kg} / \mathrm{min}$ and a predicted $\mathrm{VO}_{2 \text { peak }}<37 \%$ were optimal cut-off values for postoperative mortality (Licker et al., 2011). In another study, a $\mathrm{VO}_{2 \text { peak }}$ cut-off value of $\leq 500 \mathrm{~mL} / \mathrm{min}$ was reported to indicate a higher risk for postoperative cardiac and pulmonary complications (Epstein et al., 1993). In another study, most optimal VE/ $/ \mathrm{VCO}_{2}$-slope cut-off values for an increased risk for postoperative complications were $>35$ (Shafiek et al., 2016), while a $\mathrm{VE} / \mathrm{VCO}_{2}$-slope $>40$ was reported as a cut-off value for an increased risk for postoperative mortality (Miyazaki et al., 2018). A VO 2 peak $<19.1$ $\mathrm{mL} / \mathrm{kg} / \mathrm{min}$, measured by means of a CPET on a treadmill, was a cut-off value for an increased risk for postoperative complications (Kasikcioglu et al., 2018), whereas a VE/ $\mathrm{VCO}_{2}$-slope $\geq 34$ reflected an increased risk for postoperative mortality (Torchio et al., 2010). Participants who walked a distance $<500 \mathrm{~m}$ at the iSWT had an increased risk for cardiac and pulmonary postoperative complications (Fennelly et al., 2016). When using the preoperative 6MWT, a distance walked $<400 \mathrm{~m}$ (Irie et al., 2015) and $<500 \mathrm{~m}$ (Marjanski et al., 2015) were cut-off values for an increased risk for postoperative complications.

\section{Discussion}

This systematic review aimed to evaluate which outcome variables of pretreatment exercise tests are associated with treatment complications in patients with stage I-III NSCLC, as well as to identify cut-off values that can be used for clinical risk stratification. Results demonstrate that a wide variety of outcome variables of different preoperative exercise tests seem to be associated with postoperative complications and/or postoperative mortality. However, used exercise protocols varied widely between the studies. In addition, only a limited number of cut-off values with a moderate accuracy were provided. Publications on other treatment strategies than surgery were lacking.

The CPET is the most frequently used preoperative exercise test and mandatory in guidelines as a risk assessment tool when lung function tests values are $<80 \%$ of predicted. $\mathrm{VO}_{2 \text { peak }}$ was associated with postoperative complications and/or postoperative mortality in 18 of the 25 studies (72\%), in which a higher aerobic fitness reflected a reduced risk. Lower preoperative aerobic fitness has been shown to be associated with an increased risk for short-term and long-term postoperative complications in several other surgical populations as well (Moran et al., 2016; West et al., 2014; Moyes et al., 2013; Lee et al., 2018). Although the CPET seems to be a valuable test that is associated with postoperative complications in patients with NSCLC, accurate and consistent cut-off values to identify patients with a higher risk for complications are lacking. This means that the best method for pretreatment risk assessment based on CPET is still unclear, given the wide variety of associated outcomes and study characteristics. In the current systematic review, $\mathrm{VO}_{2 \text { peak }}$ cut-off values for an increased risk for postoperative complications ranged between $<12.8 \mathrm{~mL} / \mathrm{kg} / \mathrm{min}$ and $<19.1 \mathrm{~mL} / \mathrm{kg} / \mathrm{min}$ (Kasikcioglu et al., 2018; Licker et al., 2011). One study (Licker et al., 2011) reported a $\mathrm{VO}_{2 \text { peak }}$ cut-off value of $<58 \%$ of predicted to reflect a higher risk for cardiac complications. $\mathrm{A} \mathrm{VO}_{2 \text { peak }}$ cut-off value of $<53 \%$ of predicted was reported in the same study for a higher risk for postoperative pulmonary complications, and $<37 \%$ of predicted for a higher risk for postoperative mortality (Licker et al., 2011). Interpretation of these cut-off values is debatable, because of uncertainty concerning the used $\mathrm{VO}_{2 \text { peak }}$ references values and the poor methodological quality of studies. Several international guidelines have described a large range of $\mathrm{VO}_{2 \text { peak }}$ cut-off values between $<16 \mathrm{~mL} / \mathrm{kg} / \mathrm{min}$ and $<20 \mathrm{~mL} / \mathrm{kg} / \mathrm{min}$, 
Table 5

Cut-off values at pretreatment exercise tests for an increased risk for postoperative complications and postoperative mortality.

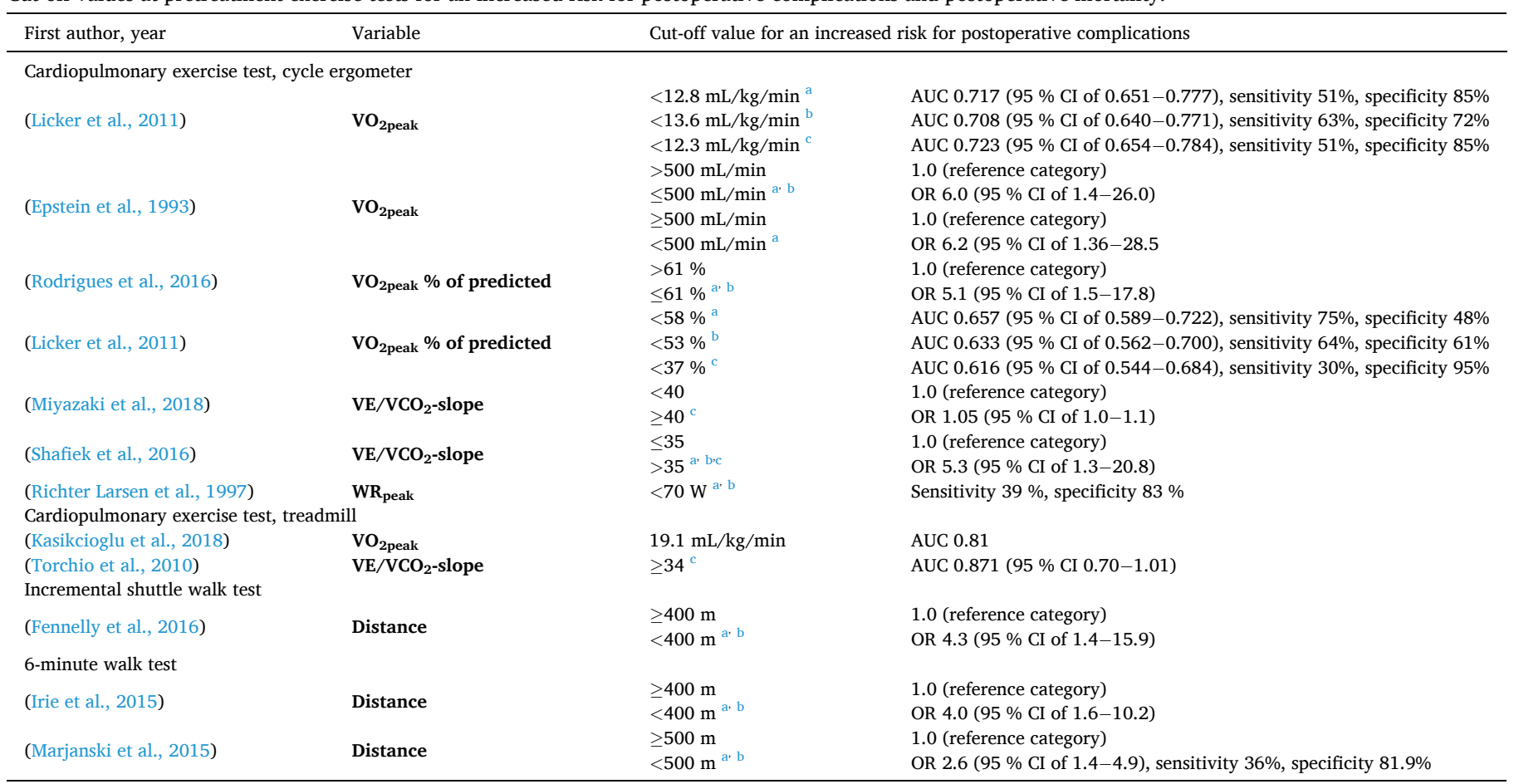

Abbreviations: $\mathrm{VE} / \mathrm{VCO}_{2}$-slope=slope describing the relationship between the minute ventilation and carbon dioxide production; $\mathrm{VO}_{2 \text { peak }}=$ oxygen uptake at peak exercise; $\mathrm{AUC}=$ area under the curve; $\mathrm{CI}=$ confidence interval; $\mathrm{WR}_{\text {peak }}=$ work rate at peak exercise; $\mathrm{ROC}=$ receiver operating characteristic.

a : cardiac complications.

b : pulmonary complications.

c : postoperative mortality.

and a $\mathrm{VO}_{2 \text { peak }}$ between $<35 \%$ and $<40 \%$ of predicted to identify patients undergoing lung resection for cancer with an increased risk for postoperative complications (Brunelli et al., 2013; Lim et al., 2010). A broad range in used cut-off values was also seen in the current review, possibly as a result of poor methodological quality and inadequate sample size of studies. $\mathrm{VO}_{2 \text { peak }}$ is a measure of aerobic fitness that requires a maximal effort of the patient, whereas the oxygen uptake at the ventilatory anaerobic threshold is a submaximal indicator of aerobic fitness that has been consistently reported to be an independent predictor of morbidity, mortality, and length of stay following major abdominal surgery (Moran et al., 2016). Nevertheless, only a limited number of studies (Fang et al., 2013; Nagamatsu et al., 2004; Bechard and Wetstein, 1987) addressed the prognostic value of the preoperative oxygen uptake at the ventilatory anaerobic threshold for postoperative outcomes in patients undergoing lung surgery. Therefore, more research is needed.

Field tests require little equipment and training prior to use (Fotheringham et al., 2015). In comparison, the CPET requires well-trained staff and relatively expensive equipment. The CPET provides a more in-depth assessment of cardiopulmonary function and gas exchange and, as described above, has been reported to predict outcome following lung cancer surgery. Unfortunately, the CPET may not always be available; making field tests an attractive alternative. However, there is only limited evidence to justify their use in the preoperative setting. Intuitively, the preoperative iSWT is more demanding than submaximal field tests and may therefore be a superior method of estimating aerobic fitness when the CPET is unavailable. Nevertheless, associations between the iSWT and postoperative complications are not covered sufficiently by study results; in only one of three studies a statistically significant association was found. Therefore, currently using the iSWT for risk-stratification seems not to be recommended. Similar to a study in abdominal surgery (Reddy et al., 2016), this systematic review demonstrated that a better performance on the preoperative SCT was associated with a lower risk for postoperative complications following lung surgery. This is in line with a previous publication, in which stair-climbing seemed to be predictive for postoperative outcomes after abdominal surgery (Reddy et al., 2016). In the current systematic review, also an association between a lower distance walked on the preoperative 6MWT and a higher risk for postoperative complications was shown. In two studies (Irie et al., 2015; Marjanski et al., 2015), 6MWT distance cut-off values of respectively $<400 \mathrm{~m}$ and $<500 \mathrm{~m}$ were associated with postoperative complications. A difference of $100 \mathrm{~m}$ in cut-off values is rather large. This is possibly a reflection of the small number of included patients. In addition, the 6MWT is susceptible to biased results, as patients can regulate their physical effort during the test which may underestimate or overestimate the results (Dourado et al., 2011). No study was found that investigated the association between the preoperative steep ramp test and postoperative complications or postoperative mortality. A previous study in adult cancer survivors demonstrated a strong correlation between steep ramp test performance and aerobic fitness $\left(\mathrm{VO}_{2 \text { peak }}\right)$ as objectively measured during the CPET (De Backer et al., 2007). Furthermore, another study in hepatic surgery demonstrated that a lower aerobic fitness, as estimated with the steep ramp test, was associated with postoperative complications (Van Beijsterveld et al., 2019). This easy-to-use short-time maximal exercise test (Bongers and Takken, 2014) might therefore also be used for preoperative risk assessment in patients with lung cancer; however, evidence is currently lacking.

To correctly interpret the results, it is essential to know that there are limitations in the included studies. A poor score on the NewcastleOttawa Scale was particularly found in articles older than ten years. This is mainly due to the non-description or incomplete description of the population, as well as the representativeness of the exposed cohort, the assessment of the outcome, and the follow-up time. There was 
considerable variation between the studies in the type of surgery, the used outcome variables of exercise tests, and the incomplete description of postoperative complications. This variation could have influenced the associations between the outcome of the exercise test, and postoperative complications or mortality. The physiological impact and risks of a segmentectomy are expected to be less than those of a pneumonectomy; therefore, in different surgical procedures it would intuitively be expected to use different relative $\mathrm{VO}_{2 \text { peak }}$ thresholds for preoperative risk stratification, depending on the extent of the surgical trauma (Licker et al., 2011).

Although studies have shown that preoperative exercise tests are associated with postoperative complications, more attention needs to be paid to which outcome variables and cut-off values of the CPET are clinically relevant, as well as to the possibility of supplementing the CPET with field tests. In an optimal situation there is a possibility of identifying high-risk patients before the start of the treatment, after which the physical performance status might be improved by prehabilitation in order to reduce a patient's risk for complications during and/or after treatment (Licker et al., 2017; Stefanelli et al., 2013).

Only surgical patients were included in this systematic review. More attention should be paid to the potential of exercise tests to predict treatment complications in patients with NSCLC who undergo other intensive treatments, such as chemoradiotherapy. Efforts should be made internationally to reach consensus on standardizing pretreatment exercise tests for accurate cut-off values in pretreatment risk stratification. In future studies, the description of postoperative complications and postoperative mortality should be used according to a standardized protocol, and consensus should be reached to use the same follow-up time regarding complications and mortality to enable pooling of study results. Currently, the evidence of field tests to predict treatment complications is weaker than for the CPET. In addition, research regarding the prognostic values of pretreatment field tests for treatment complications is of poor quality, which underlines the need for high-quality research using standardized field exercise test protocols.

\section{Conclusion}

A better performance of patients on preoperative exercise tests, especially a higher aerobic fitness as measured by the CPET, is associated with a lower risk for postoperative complications in patients with NSCLC. However, it is difficult to provide recommendations for pretreatment exercise tests to predict the risk of treatment complications due to a lack of accurate test-specific cut-off values. Additionally, recommendations for the use of field tests are difficult due to heterogeneity in tests, protocols, and used outcome measures in the current literature. Therefore, standardizing pretreatment exercise test protocols is eminent and more attention needs to be paid to which outcome variables and cutoff values of pretreatment exercise tests are clinically relevant. In addition, further research is needed concerning the ability of pretreatment exercise tests to accurately identify patients who have an increased risk for treatment complications across all curative NSCLC treatment options. This is important, as especially these high-risk patients might benefit from interventions to improve their physical performance status before treatment initiation.

\section{Author contributions}

The responsibility for the content of the manuscript is taken by MV. Both MV and RF have made substantial contributions to the acquisition of data, analysis and interpretation. All authors contributed to the interpretation and critical revision of the contents of the manuscript, provided approval for the current manuscript version, and agreed to be accountable for all aspects of the work and remaining questions.

\section{Funding}

This study was supported by an unconditional research grant (E.17.31.033-6) from the Research and Innovation fund VieCuri (Fonds Wetenschap en Innovatie VieCuri, Venlo, the Netherlands). This research did not receive any specific grants from funding agencies in the public, commercial, or not-for-profit sectors.

\section{Declaration of Competing Interest}

The authors report no declarations of interest.

\section{Acknowledgements}

The authors like to thank librarian Quinten de Bakker for assisting in constructing the search strategy and Dr. Frits van Osch for supporting in how to assess the methodological quality.

\section{References}

Bagg, L.R., 1984. The 12-min walking distance; its use in the pre-operative assessment of patients with bronchial carcinoma before lung resection. Respiration 46 (4), 342-345.

Ambrosino, N., 1999. Field tests in pulmonary disease. Thorax 54 (3), 191-193.

Auperin, A., Le Pechoux, C., Rolland, E., Curran, W.J., Furuse, K., Fournel, P., et al., 2010. Meta-analysis of concomitant versus sequential radiochemotherapy in locally advanced non-small-cell lung cancer. J. Clin. Oncol. 28 (13), 2181-2190.

Bechard, D., Wetstein, L., 1987. Assessment of exercise oxygen consumption as preoperative criterion for lung resection. Ann. Thorac. Surg. 44 (4), 344-349.

Bolliger, C.T., Wyser, C., Roser, H., Soler, M., Perruchoud, A.P., 1995. Lung scanning and exercise testing for the prediction of postoperative performance in lung resection candidates at increased risk for complications. Chest 108 (2), 341-348.

Bongers, B.C., Takken, T., 2014. The paediatric version of the steep ramp test. J. Physiother. 60 (2), 113.

Brunelli, A., Al Refai, M., Monteverde, M., Borri, A., Salati, M., Fianchini, A., 2001. Stair climbing test predicts cardiopulmonary complications after lung resection. Chest 121 (4), 1106-1110.

Brunelli, A., Charloux, A., Bolliger, C.T., Rocco, G., Sculier, J.P., Varela, G., et al., 2009a. ERS/ESTS clinical guidelines on fitness for radical therapy in lung cancer patients (surgery and chemo-radiotherapy). Eur. Respir. J. 34 (1), 17-41.

Brunelli, A., Belardinelli, R., Refai, M., Salati, M., Socci, L., Pompili, C., et al, 2009b. Peak oxygen consumption during cardiopulmonary exercise test improves risk stratification in candidates to major lung resection. Chest 135 (5), 1260-1267.

Brunelli, A., Kim, Aw, Berger, Ki, Addrizzo-Harris, Dj., 2013. Physiologic evaluation of the patient with lung cancer being considered for resectional surgery: diagnosis and management of lung cancer, 3rd ed: American College of Chest Physicians evidencebased clinical practice guidelines. Chest 143 (5 Suppl) e166S-e90S.

Brutsche, M.H., Spiliopoulos, A., Bolliger, C.T., Licker, M., Frey, J.G., Tschopp, J.M., 2000. Exercise capacity and extent of resection as predictors of surgical risk in lung cancer. Eur. Respir. J. 15 (5), 828-832.

Campione, A., Terzi, A., Bobbio, M., Rosso, G.L., Scardovi, A.B., Feola, M., 2010. Oxygen pulse as a predictor of cardiopulmonary events in lung resection. Asian Cardiovasc. Thorac. Ann. 18 (2), 147-152.

Cavalheri, V., Jenkins, S., Cecins, N., Gain, K., Hill, K., 2016. Comparison of the six minute walk test with a cycle-based cardiopulmonary exercise test in people following curative intent treatment for non-small cell lung cancer. Chron. Respir. Dis. 13 (2), 118-127.

Dales, R.E., Dionne, G., Leech, J.A., Lunau, M., Schweitzer, I., 1993. Preoperative prediction of pulmonary complications following thoracic surgery. Chest 104 (1), 155-159.

David Moher, A.L., Tetzlaff, Jennifer, Altman, Douglas G., 2009. Preferred reporting items for systematic reviews and meta-analyses: the PRISMA statement. J. Clin. Epidemiol. 62, 1006-1012, 2009.

De Backer, I.C., Schep, G., Hoogeveen, A., Vreugdenhil, G., 2007. Kester AD, van Breda E. Exercise testing and training in a cancer rehabilitation program: the advantage of the steep ramp test. Arch. Phys. Med. Rehabil. 88 (5), 610-616.

Dong, J., Mao, Y., Li, J., He, J., 2017. Stair-climbing test predicts postoperative cardiopulmonary complications and hospital stay in patients with non-small cell lung cancer. Med. Sci. Monit. 23, 1436-1441.

Dourado, V., Vidotto, M.C., Guerra, R.L., 2011. Reference equations for the performance of healthy adults on field walking tests. J. Bras. Pneumol. 37 (5), 607-614.

Driessen, Ej, Bootsma, Gp, Hendriks, Le, van den Berkmortel, Fw, Bogaarts, Ba, van Loon, Jg, et al., 2016. Stage III non-small cell lung cancer in the elderly: patient characteristics predictive for tolerance and survival of chemoradiation in daily clinical practice. Radiother. Oncol. 121 (1), 26-31.

Eberhardt, W.E., De Ruysscher, D., Weder, W., Le Pechoux, C., De Leyn, P., Hoffmann, H., et al., 2015. 2nd ESMO consensus conference in lung cancer: locally advanced stage III non-small-cell lung cancer. Ann. Oncol. 26 (8), 1573-1588. 
Epstein, S.K., Faling, L.J., Daly, B.D.T., Celli, B.R., 1993. Predicting complications after pulmonary resection: preoperative exercise testing vs a multifactorial cardiopulmonary risk index. Chest 104 (3), 694-700.

Erdoğan, Y., Günay, E., Ergün, P., Kaymaz, D., Temiz, G., Karaoğlanoğlu, N., 2013. Can exercise capacity assessed by the shuttle walk test predict the development of postoperative complications in patients with lung cancer? Tuberkuloz ve Toraks. 61 (1), 28-32.

Fang, Y., Ma, G., Lou, N., Liao, W., Wang, D., 2013. Preoperative maximal oxygen uptake and exercise-induced changes in pulse oximetry predict early postoperative respiratory complications in lung cancer patients. Scand. J. Surg. 103 (3), 201-208.

Fennelly, J., Potter, L., Pompili, C., Brunelli, A., 2016. Performance in the shuttle walk test is associated with cardiopulmonary complications after lung resections. J. Thorac. Dis. 9 (3), 789-795.

Fotheringham, I., Meakin, G., Punekar, Y.S., Riley, J.H., Cockle, S.M., Singh, S.J., 2015. Comparison of laboratory- and field-based exercise tests for COPD: a systematic review. Int. J. Chron. Obstruct. Pulmon. Dis. 10, 625-643.

Granger, C.L., Denehy, L., Parry, S.M., Martin, J., Dimitriadis, T., Sorohan, M., et al., 2015. Which field walking test should be used to assess functional exercise capacity in lung cancer? An observational study. BMC Pulm. Med. 15, 89.

Ha, D., Choi, H.K., McCarthy, K., Minai, O.A., Raymond, D., Mazzone, P.J., 2013. Association of impaired heart rate recovery with cardiopulmonary complications after lung resection surgery. Am. J. Respir. Crit. Care Med. 187.

Holden, D.A., Rice, T.W., Stelmach, K., Meeker, D.P., 1992. Exercise testing, 6-min walk, and stair climb in the evaluation of patients at high risk for pulmonary resection. Chest 102 (6), 1774-1779.

Holland, A.E., Dowman, L., Fiore Jr., J., Brazzale, D., Hill, C.J., McDonald, C.F., 2014. Cardiorespiratory responses to 6-minute walk test in interstitial lung disease: not always a submaximal test. BMC Pulm. Med. 14, 136.

Irie, M., Nakanishi, R., Yasuda, M., Fujino, Y., Hamada, K., Hyodo, M., 2015. Risk factors for short-term outcomes after thoracoscopic lobectomy for lung cancer. Eur. Respir. J. 48 (2), 495-503.

Janssen-Heijnen, M.L., Smulders, S., Lemmens, V.E., Smeenk, F.W., van Geffen, H.J., Coebergh, J.W., 2004. Effect of comorbidity on the treatment and prognosis of elderly patients with non-small cell lung cancer. Thorax 59 (7), 602-607.

Jemal, A., Bray, F., Center, M.M., Ferlay, J., Ward, E., Forman, D., 2011. Global cancer statistics. CA Cancer J. Clin. 61 (2), 69-90.

Jones, L.W., Eves, N.D., Mackey, J.R., Peddle, C.J., Haykowsky, M., Joy, A.A., et al., 2007. Safety and feasibility of cardiopulmonary exercise testing in patients with advanced cancer. Lung Cancer 55 (2), 225-232.

Kasikcioglu, E., Toker, A., Tanju, S., Arzuman, P., Kayserilioglu, A., Dilege, S., et al., 2018. Oxygen uptake kinetics during cardiopulmonary exercise testing and postoperative complications in patients with lung cancer. Lung Cancer 66 (1), $85-88$.

Lee, C.H.A., Kong, J.C., Ismail, H., Riedel, B., Heriot, A., 2018. Systematic review and meta-analysis of objective assessment of physical fitness in patients undergoing colorectal cancer surgery. Dis. Colon Rectum 61 (3), 400-409.

Licker, M., Schnyder, J.M., Frey, J.G., Diaper, J., Cartier, V., Inan, C., et al., 2011. Impact of aerobic exercise capacity and procedure-related factors in lung cancer surgery. Eur. Respir. J. 37 (5), 1189-1198.

Licker, M., Karenovics, W., Diaper, J., Frésard, I., Triponez, F., Ellenberger, C., et al., 2017. Short-term preoperative high-intensity interval training in patients awaiting lung cancer surgery: a randomized controlled trial. J. Thorac. Oncol. 12 (2), 323-333.

Lim, E., Baldwin, D., Beckles, M., Duffy, J., Entwisle, J., Faivre-Finn, C., et al., 2010. Guidelines on the radical management of patients with lung cancer. Thorax 65 (Suppl 3), 1-27.

Marjanski, T., Wnuk, D., Bosakowski, D., Szmuda, T., Sawicka, W., Rzyman, W., 2015. Patients who do not reach a distance of $500 \mathrm{~m}$ during the 6-min walk test have an increased risk of postoperative complications and prolonged hospital stay after lobectomy. Eur. J. Cardiothorac. Surg. 47 (5), e213-9.

Miyazaki, T., Brunelli, A., Dimesh, P., Callster, M., Franks, K., Nagayasu, T., 2018. Minute ventilation-to-carbon dioxide slope is associated with early and long term survivals following anatomical pulmonary resection. J. Thorac. Oncol. 13 (10), S645.

Moran, J., Wilson, F., Guinan, E., McCormick, P., Hussey, J., Moriarty, J., 2016. Role of cardiopulmonary exercise testing as a risk-assessment method in patients undergoing intra-abdominal surgery: a systematic review. Br. J. Anaesth. 116 (2), 177-191.

Moyes, L.H., McCaffer, C.J., Carter, R.C., Fullarton, G.M., Mackay, C.K., Forshaw, M.J., 2013. Cardiopulmonary exercise testing as a predictor of complications in oesophagogastric cancer surgery. Ann. R. Coll. Surg. Engl. 95 (2), 125-130.

Nagamatsu, Y., Shima, I., Hayashi, A., Yamana, H., Shirouzo, K., Ishitake, T., 2004. Preoperative spirometry versus expired gas analysis during exercise testing as predictors of cardiopulmonary complications after lung resection. Surg. Today 34 (2), 107-110.

Nagamatsu, Y., Sueyoshi, S., Tsubuku, T., Kawasaki, M., Akagi, Y., 2015. Predicting postoperative exercise capacity after major lung resection. Surg. Today 45 (12), 1501-1508.

Nakagawa, T., Tomioka, Y., Toyazaki, T., Gotoh, M., 2018. Association between values of preoperative 6-min walk test and surgical outcomes in lung cancer patients with decreased predicted postoperative pulmonary function. Gen. Thorac. Cardiovasc. Surg. 66 (4), 220-224.

Netherlands Cancer Registry, 2016. Incidence of Non-small Cell Lung Cancer in the Netherlands According to Type of Histology (1990-2019) (cited 2020): Comprehensive Cancer center the Netherlands (IKNL). Available from:http:// cijfersoverkankernl/selecties/Dataset 1/img576b9713d1fe6.
Ni, H.J., Pudasaini, B., Yuan, X.T., Li, H.F., Shi, L., Yuan, P., 2017. Exercise training for patients pre- and postsurgically treated for non-small cell lung cancer: a systematic review and meta-analysis. Integr. Cancer Ther. 16 (1), 63-73.

Nikolic, I., Majeric-Kogler, V., Plavec, D., Maloca, I., Slobodnjak, Z., 2007. Stairs climbing test with pulse oximetry as predictor of early postoperative complications in functionally impaired patients with lung cancer and elective lung surgery: prospective trial of consecutive series of patients. Croat. Med. J. 49 (1), 50-57.

Pate, P., Tenholder, Mf, Griffin, Jp, Eastridge, Ce, Weiman, Ds., 1996. Preoperative assessment of the high-risk patient for lung resection. Ann. Thorac. Surg. 61 (5), 1494-1500.

Perrotta, F., Cennamo, A., Cerqua, F.S., Stefanelli, F., Bianco, A., Musella, S., et al., 2019. Effects of a high-intensity pulmonary rehabilitation program on the minute ventilation/carbon dioxide output slope during exercise in a cohort of patients with COPD undergoing lung resection for non-small cell lung cancer. J. Bras. Pneumol. 45 (6), e20180132.

Postmus, P.E., Kerr, K.M., Oudkerk, M., Senan, S., Waller, D.A., Vansteenkiste, J., et al., 2017. Early and locally advanced non-small-cell lung cancer (NSCLC): ESMO clinical practice guidelines for diagnosis, treatment and follow-up. Ann. Oncol. 28 (4), 1-21.

Reddy, S., Contreras, C.M., Singletary, B., Bradford, T.M., Waldrop, M.G., Mims, A.H., et al., 2016. Timed stair climbing is the single strongest predictor of perioperative complications in patients undergoing abdominal surgery. J. Am. Coll. Surg. 222 (4), 559-566.

Refai, M., Pompili, C., Salati, M., Xiumè, F., Sabbatini, A., Brunelli, A., 2014. Can maximal inspiratory and expiratory pressures during exercise predict complications in patients submitted to major lung resections? A prospective cohort study. Eur. J. Cardio-thoracic Surg. 45 (4), 665-670.

Richter Larsen, K., Svendsen, U.G., Milman, N., Brenoe, J., Petersen, B.N., 1997. Exercise testing in the preoperative evaluation of patients with bronchogenic carcinoma. Eur. Respir. J. 10 (7), 1559-1565.

Rodrigues, F., Grafino, M., Faria, I., Pontes da Mata, J., Papoila, A.L., Felix, F., 2016. Surgical risk evaluation of lung cancer in COPD patients - a cohort observational study. Rev. Port. Pneumol. 22 (5), 266-272.

Roman, M.A., Koelwyn, G.J., Eves, N.D., Hornsby, W.E., Watson, D., Herndon Ii, J.E., et al., 2014. Comparison of performance status with peak oxygen consumption in operable patients with non-small-cell lung cancer. Respirology 19 (1), 105-108.

Shafiek, H., Valera, J.L., Togores, B., Torrecilla, J.A., Sauleda, J., Cosio, B.G., 2016. Risk of postoperative complications in chronic obstructive lung diseases patients considered fit for lung cancer surgery: beyond oxygen consumption. Eur. J. Cardiothorac. Surg. 50 (4), 772-779.

Smith, T.P., Kinasewitz, G.T., Tucker, W.Y., Spillers, W.P., George, R.B., 1984. Exercise capacity as a predictor of post-thoracotomy morbidity. Am. Rev. Respir. Dis. 129 (5), 730-734.

Stefanelli, F., Meoli, I., Cobuccio, R., Curcio, C., Amore, D., Casazza, D., et al., 2013. High-intensity training and cardiopulmonary exercise testing in patients with chronic obstructive pulmonary disease and non-small-cell lung cancer undergoing lobectomy. Eur. J. Cardiothorac. Surg. 44 (4), e260-5.

Toker, A., Ziyade, S., Bayrak, Y., Tanju, S., Senturk, M., Dilege, S., et al., 2007. Prediction of cardiopulmonary morbidity after resection for lung cancer: stair climbing test complications after lung cancer surgery. Thorac. Cardiovasc. Surg. 55 (4), 253-256.

Torchio, R., Guglielmo, M., Giardino, R., Ardissone, F., Ciacco, C., Gulotta, C., et al., 2010. Exercise ventilatory inefficiency and mortality in patients with chronic obstructive pulmonary disease undergoing surgery for non-small-cell lung cancer. Eur. J. Cardiothorac. Surg. 38 (1), 14-19.

Vainshelboim, B., 2019. Cochrane Handbook for Systematic Reviews of Interventions, 2nd edition. John Wiley \& Sons, Chichester (UK).

Van Beijsterveld, C.A., Bongers, B.C., Den Dulk, M., Van Kuijk, S.M.J., Dejong, K.C.H., Van Meeteren, N.L.U., 2019. The association between preoperative physical functioning and short-term postoperative outcomes: a cohort study of patients undergoing elective hepatic resection. HPB (Oxford) 21 (10), 1362-1370.

Vansteenkiste, J., Crino, L., Dooms, C., Douillard, J.Y., Faivre-Finn, C., Lim, E., et al., 2014. 2nd ESMO consensus conference on lung cancer: early-stage non-small-cell lung cancer consensus on diagnosis, treatment and follow-up. Ann. Oncol. 25 (8), $1462-1474$.

Varela, G., Novoa, N., Ballesteros, E., Oliveira, R., Jimenez, M.F., Esteban, P.A., et al., 2009. Results of a simple exercise test performed routinely to predict postoperative morbidity after anatomical lung resection. Eur. J. Cardiothorac. Surg. 37 (3), 521-524.

Vargas Fajardo Mdel, C., Novoa Valentin, N.M., Jimenez Lopez, M.F., Ramos Gonzalez, J., Varela Simo, G., 2014. An alternative method for predicting the risk of postoperative complications in lung resection. Arch. Bronconeumol. 50 (3), 87-92.

Villani, F., Busia, A., 2004. Preoperative evaluation of patients submitted to pneumonectomy for lung carcinoma: role of exercise testing. Tumori 90 (4), 405-409.

Villani, F., De Maria, P., Busia, A., 2003. Exercise testing as a predictor of surgical risk after pneumonectomy for bronchogenic carcinoma. Respir. Med. 97 (12), 1296-1298.

Warner, A., Dahele, M., Hu, B., Palma, D.A., Senan, S., Oberije, C., et al., 2016. Factors associated with early mortality in patients treated with concurrent chemoradiation therapy for locally advanced non-small cell lung cancer. Int. J. Radiat. Oncol. Biol. Phys. 94 (3), 612-620.

Wells, G.A.S.B., O'Connell, D., Peterson, J., Welch, V., Losos, M., Tugwell, P., 2013. The Newcastle-Ottawa Scale (NOS) for Assessing the Quality of Nonrandomised Studies in Meta-Analyses. ON-Ottawa Hospital Research Institute, Ottawa.

West, M.A., Lythgoe, D., Barben, C.P., Noble, L., Kemp, G.J., Jack, S., et al., 2014. Cardiopulmonary exercise variables are associated with postoperative morbidity 
after major colonic surgery: a prospective blinded observational study. Br. J. Anaesth. 112 (4), 665-671.

Win, T., Jackson, A., Groves, A.M., Wells, F.C., Ritchie, A.J., Munday, H., et al., 2004 Relationship of shuttle walk test and lung cancer surgical outcome. Eur. J. Cardiothoracic Surg. 26 (6), 1216-1219.
Win, T., Jackson, A., Sharples, L., Groves, A.M., Wells, F.C., Ritchie, A.J., et al., 2005 Cardiopulmonary exercise tests and lung cancer surgical outcome. Chest 127 (4), 1159-1165.

Yakal, S., Sofyali, S., Ozkan, B., Yildiz, S., Toker, A., Kasikcioglu, E., 2018. Oxygen uptake efficiency slope and prediction of post-operative morbidity and mortality in patients with lung cancer. Lung 196 (2), 255-262. 